\title{
First Passage Time Computation in Tagged GSPNs with Queue Places
}

\author{
Gianfranco Balbo $^{1}$, Marco Beccuti ${ }^{1}$, Massimiliano \\ De Pierro ${ }^{1}$ and Giuliana Franceschinis ${ }^{2}$ \\ ${ }^{1}$ Dipartimento di Informatica Università degli Studi di Torino, Italy \\ ${ }^{2}$ Dipartimento di Informatica Univ. del Piemonte Orientale, Alessandria, Italy \\ Email:balbo@di.unito.it,beccuti@di.unito.it,depierro@di.unito.it, \\ giuliana.franceschinis@di.unipmn.it
}

\begin{abstract}
This paper presents an extension of the Generalized Stochastic Petri Net (GSPN) formalism that enables the computation of first passage time distributions. The tagged customer technique typical of queuing networks is adapted to the GSPN context by providing a formal definition and an automatic computation of the groups of tokens that can be identified as customers, i.e. classes of homogeneous entities behaving in a similar manner. Passage times are identified through the concept of events that correspond to the firing of transitions placed at the boundaries of a subnet. The extended model obtained with this specifications is translated into an ordinary GSPN by isolating a customer from the group and highlighting its path through the net thus obtaining a representation suited for the passage time analysis. Proofs are provided to show the equivalence between these models with respect to their steady state distributions. An important and original aspect treated in this paper is the possibility of specifying several scheduling policies of tokens at places, an information not present in ordinary GSPN models, but that is vital for the precise computation of first passage time distributions as shown by a few results computed for a simple Flexible Manufacturing application.
\end{abstract}

Keywords: Fist passage time, Petri Nets, Tagged customer

\section{INTRODUCTION}

Generalized Stochastic Petri Nets (GSPNs) [1] are a modeling formalism very effective in the representation and evaluation of many real systems. Among the advantages of modeling with GSPNs are the precision and the expressive power of their constructs together with their well defined time semantics that allow the direct definition of the Markov chains representing their underlying stochastic processes used to compute many classical performance indices. The standard analysis of GSPN models amounts to the computation of their transient and stationary state probability distributions that are used to assess their dynamics.

This paper reports on an effort to augment the modeling power of GSPNs providing definitions, constructs and methodologies useful for an easy and straightforward computation of passage times among states of the model. Our work is both an extension and a refinement of that of [2] which uses the tagged customer approach to assess the behavior of GSPN models with "customer-centric" performance measures.

As observed in [2], prior work on this type of approach in the context of GSPN analysis is very limited. Most of the papers that present the use of the tagged customer method, that is typical of queuing theory [3], in models developed with Petri net [4], Process Algebra [5] or other formalisms for the specification of Markov Chains [6], require a manual intervention on the original model that is often difficult to perform. Computing passage times between sets of states in Markov chains is a well known problem with limited theoretical difficulties. Disregarding for the moment the computational complexity of the solution in the case of very large models for which specific and powerful tools exist $[6,7]$, the actual difficulty of this approach consists in mapping the situations that arise when the tagged customer enters (leaves) a specific portion of the model onto the corresponding set of Markov chain states that represent the boundaries for the passage time computations. Driven by application purposes, in $[2,8]$ the notion of "customer" is informally brought within the GSPN's domain making it useful for the analysis of specific cases, but leaving uncertain what to do in other situations when models generate peculiar behaviors that require more precise definitions.

In this paper we will recall the concept of "tagged token" in GSPN introduced in [2] and provide proper and precise methods for defining the beginning and the end of passage times at the GSPN level. 
Tokens in GSPNs cannot be interpreted, in general, as entities that travel throughout the models, but are instead indistinguishable quantities consumed and generated at transition firing instants. The idea of selecting one of these tokens to make it "tagged" and of following it throughout the net is not a trivial task, unless certain structural properties of the net are exploited. Our proposal envisions that invariant properties which correspond to conservation conditions within the net ( $\mathrm{p}$-semiflows) are computed first in order to identify places where tokens with indistinguishable behavior are preserved. Tokens of this type can be treated similarly to the customers of QN models and can thus be tagged by the modeler in order to compute the distribution of the time they take to travel between points of the net.

The TGSPN (Tagged Generalized Stochastic Petri Nets) formalism proposed in this paper extends classical GSPNs with primitives that allow to specify the input/output interfaces of submodels that are useful for the passage time computations as well as the p-invariants which identify classes of tokens with undistinguishable behaviors.

The intersection between these p-invariants and the sets of places of the submodels identifies the places where the tagged customer can be found and thus the portion of GSPN that needs to be manipulated in order to allow the first-passage-time analysis.

The tagged token approach allows a richer analysis of the model, but has an obvious computational cost due to the larger state space that it induces. Moreover, the introduction of the tagged customer makes the policies used to manage the tokens in the input places of a transition relevant for the performance measures obtained with this analysis and it must be explicitly addressed. The way in which we account for this problem builds on the experience of embedding queuing policies in the input place of a timed transition of a GSPN that led to the proposal of Queuing Petri Nets (QPNs) [9, 10]. We provide compact and parametric representations of the different input queuing policies, specifically tailored to the goal of tracking the tagged token position in the queue. The difference is that in QPNs the special queuing places embed the queue, the server and a sort of output buffer for the tokens that have already received service that we instead exclude from our representations.

The main contributions of this paper are thus (1) a precise extension of the GSPN formalism to guide the identification of the tagged tokens and to support the first passage time analysis; (2) the introduction of a number of queuing policies that can be properly analyzed.

The article, which is an extended and revised version of [11], is organized as follows: Sec. 2 is a brief introduction to the GSPN formalism; using the GSPN formalism, a model inspired from a Flexible Manufacturing System (FMS) application is described that will be used throughout the paper as a "running" example; Sec. 3 introduces the method for identifying "taggable" classes of tokens and provides the TGSPN formal definition; Sec. 4 introduces the method used to specify the measures of interest at the net level; Sec. 5 provides automatic rules which translate the TGSPN into a GSPN model in order to apply the analysis and Sec. 6 extends the specification with queue policies; Sec. 7 illustrates the sequence of actions that implement the analysis procedure, discussing each individual step and specifically the way in which the GSPN generated in the previous step is used; Sec. 8 presents a few numerical results chosen to show the relevance of the new formalism and the importance of the analysis that can be performed with the tagged token approach; Sec. 9 compares the proposed approach with other works from the literature. Finally, Sec. 10 concludes this work with a brief discussion of the results that have been obtained and with the identification of possible directions for future research on this topics.

\section{THE GSPN FORMALISM}

Generalized Stochastic Petri Nets (GSPNs) extend classical Place/Transition (Petri) nets with timing specifications. GSPNs are used in many application fields for the validation and evaluation of distributed systems characterized by concurrency, synchronization and congestion. A detailed introduction to the formalism and to its applications can be found in [1]. Here we recall the notation and the basic definitions that are used in the rest of the paper.

Definition 2.1. A GSPN system is a tuple $\left(P, T, I^{-}, I^{+}, H, \Pi, \mathrm{w}, \mathbf{m}_{0}\right)$ where:

- $P=\left\{p_{i}\right\}$ is a finite and non empty set of places.

- $T=E \cup Z$ is a finite and non empty set of transitions.

$E$ is the set of timed transitions which fire with a random delay characterized by a negative exponential probability distribution; each of these transitions can be either of single-server, n-server, or $\infty$-server type. $Z$ is the set of immediate transitions which fire in zero time;

$P \cap T=\emptyset$ and $Z \cap E=\emptyset$.

- $I^{-}, I^{+}, H: T \times P \rightarrow \mathbb{N}$ are the input, output, and inhibition functions that define the arcs of the net and that specify their multiplicities.

- $\Pi: T \rightarrow \mathbb{N}$ is the (absolute) priority function. $\forall t \in E, \Pi(t)=0, \forall t \in Z, \Pi(t)>0$.

- $w: \mathbb{N}^{P} \times T \rightarrow \mathbb{R}$ is a (possibly marking dependent) function that assigns to each transition of the net:

(1) the rate of a negative exponential distribution of the firing delay when transition $t \in E$;

(2) the weight (used in case of probabilistic choices) when transition $t \in Z$.

- $\mathbf{m}_{\mathbf{0}}: P \rightarrow \mathbb{N}$ is a multiset on $P$ representing the initial marking of the net. 
A marking $\mathbf{m}$ (or state) of a GSPN is a multiset on $P$. The set of input places of transition $t$ (also referred to as the preset of $t$ ) is denoted $\bullet t$, the set of output places (or postset) of $t$ is denoted $t^{\bullet}$, and the set of inhibitor places of $t$ is denoted ${ }^{\circ} t$, are defined as follows:

$\bullet t:=\left\{p \in P \mid I^{-}(t, p)>0\right\}$

$t^{\bullet}:=\left\{p \in P \mid I^{+}(t, p)>0\right\}$,

${ }^{\circ} t:=\{p \in P \mid H(t, p)<\infty\}$.

A similar dot notation and similar definitions hold for the places of the net.

A transition $t$ has concession at marking $\mathbf{m}$ iff $I^{-}(t, p) \leq \mathbf{m}(p)<H(t, p), \forall p \in P$, where $\mathbf{m}(p)$ represents the number of tokens in place $p$ in marking $\mathbf{m}$. A transition $t$ is enabled in marking $\mathbf{m}$ if no higher priority transitions have concession in that same marking. The set of transitions enabled in $\mathbf{m}$ is denoted $\mathcal{E}(\mathbf{m})$. Enabled transitions may occur, or fire.

The occurrence of transition $t$ in marking $\mathbf{m}$ yields a marking $\mathbf{m}^{\prime}=\mathbf{m}+I^{+}(t)-I^{-}(t)$. Marking $\mathbf{m}^{\prime}$ is said to be reachable from $\mathbf{m}$ because of the firing of $t$ and is denoted by $\mathbf{m}[t\rangle \mathbf{m}^{\prime}$. The occurrence of a sequence $\sigma$ of transitions enabled at $\mathbf{m}$ and yielding $\mathbf{m}^{\prime}$ is denoted similarly: $\mathbf{m}[\sigma\rangle \mathbf{m}^{\prime}$.

The enabling degree of an enabled transition $t$ in $\mathbf{m}$ (denoted $\operatorname{ed}(t, \mathbf{m})=k$ ) is the greatest natural $k$ such that $k I^{-}(t, p) \leq \mathbf{m}(p) \forall p$. In a given marking $\mathbf{m}$, a n-server timed transition $t$ carries on many activities in parallel, whose number is given by $\min (n, e d(t, \mathbf{m}))$.

A marking where no immediate transitions are enabled is called tangible, otherwise vanishing. In GSPN models, the tangible markings which are reachable from a given initial tangible marking $\mathbf{m}_{0}$ form the Tangible Reachability Set (TRS) and are the states of an underlying Markov Chain (MC) describing the temporal behavior of the system. Tangible and vanishing markings which are reachable from a given initial marking $\mathbf{m}_{0}$, connected by arcs representing the transition firings that allow to move from one marking to the next one, form the Reachability Graph (RG). The Tangible Reachability Graph is a structure which includes the tangible states only, and whose arcs represent sequences of one timed transition firing followed by zero or more immediate transition firings, leading to the next tangible marking. The transition rates among TRG states are defined in terms of the rates associated with timed transitions and of the weights of the immediate ones. The immediate transition weights are used to decide which among several concurrently enabled transitions must fire first. This allows to probabilistically solve possible conflicts. In this paper we assume that probabilities are derived by renormalizing (in each marking) the weights of all the enabled immediate transitions in order to obtain a probability distribution on such set of transitions. Other approaches are possible, based on locally defined net-level weights (defined by the user on the basis of structurally detected potential conflicts) renormalized in each marking on the basis of the set of enabled transitions and on the actual conflicts [1, 12].

A p-flow $\mathbf{f}$ is a $|P|$-component vector, solution of the system of linear equations $\mathbf{f} \cdot C=\mathbf{0}$, where $C$ is the GSPN incidence matrix $\left(C[i, j]=I^{+}\left(t_{i}, p_{j}\right)-I^{-}\left(t_{i}, p_{j}\right)\right)$ and · is the scalar product. A solution $\mathbf{f}$ whose coefficients are natural and non-negative numbers is called a p-semiflow. The support of a p-semiflow is the set of places corresponding to the positive components of the semiflow itself. In our work, we are interested in minimal p-semiflows: a minimal p-semiflow is such that it can not be obtained as a positive linear combination of other p-semiflows, which also means that there are no other semiflows whose supports are properly included in the support of the minimal one. When the positive components of a p-semiflow are all equal to one, its interpretation is particularly simple: in fact it means that the number of tokens distributed on its support is unaffected (remains unchanged) by the firing of any transition of the net. In general the p-semiflows are related to invariant laws of the net, in particular to place invariants. Formally, this property is captured by the observation that for each p-semiflow of the GSPN $\mathbf{f} \cdot \mathbf{m}=\mathbf{f} \cdot \mathbf{m}_{0}=K$ (a constant called place invariant) for any marking $\mathbf{m}$ reachable from $\mathbf{m}_{0}$. The sum notation $\sum_{i} \mathbf{f}[i] \cdot p_{i}$ is often used to denote the p-semiflow $\mathbf{f}$.

\subsection{The running example - a FMS}

Fig. 1 shows a GSPN model of a Flexible Manufacturing System (FMS). This FMS comprises four manufacturing stations, from $\mathrm{M}_{1}$ through $\mathrm{M}_{4}$, where two of them, $\mathrm{M}_{2}$ and $\mathrm{M}_{3}$, can fail. Raw parts are loaded on suitable pallets at the Load/Unload station represented by the pair - place $p_{0}$ and transition $T_{0}$ - and are then manufactured, being sequentially brought to the four machines. When the manufacturing is completed, the finished part is replaced on the pallet by a raw one and the cycle starts again. Machine $\mathrm{M}_{2}$ has a set of local tools enabling a faster replacement of faulty machine components in order to continue its operation without the immediate intervention of a repairman. When a tool breaks down and no more spare parts are available, the intervention of a repairman for maintenance is required, which repairs all the down tools. Machine $\mathrm{M}_{3}$ does not have local tools and any of its failures must be handled by a repairman. A set of repairmen (initially located in place $p_{19}$ ) operate on failed machines as illustrated in the lower part of the picture. They cycle between vacation and repair periods. Upon return from a vacation $\left(T_{9}\right)$ a repairman checks if a machine has failed $\left(t_{5}, t_{6}\right)$ and in such case starts a repair activity $\left(T_{7}, T_{8}\right)$, otherwise goes back to vacation $\left(t_{7}\right)$. After the repairman ends working on a failed machine, he takes a rest $\left(T_{10}\right)$ before starting a new cycle. Priority is given to machine $\mathrm{M}_{3}$ when a repairman sees that both $\left(\mathrm{M}_{2}\right.$ and $\mathrm{M}_{3}$ ) failed.

This net has twelve minimal p-semiflows, illustrated in Tab. 1, related to different entities represented 


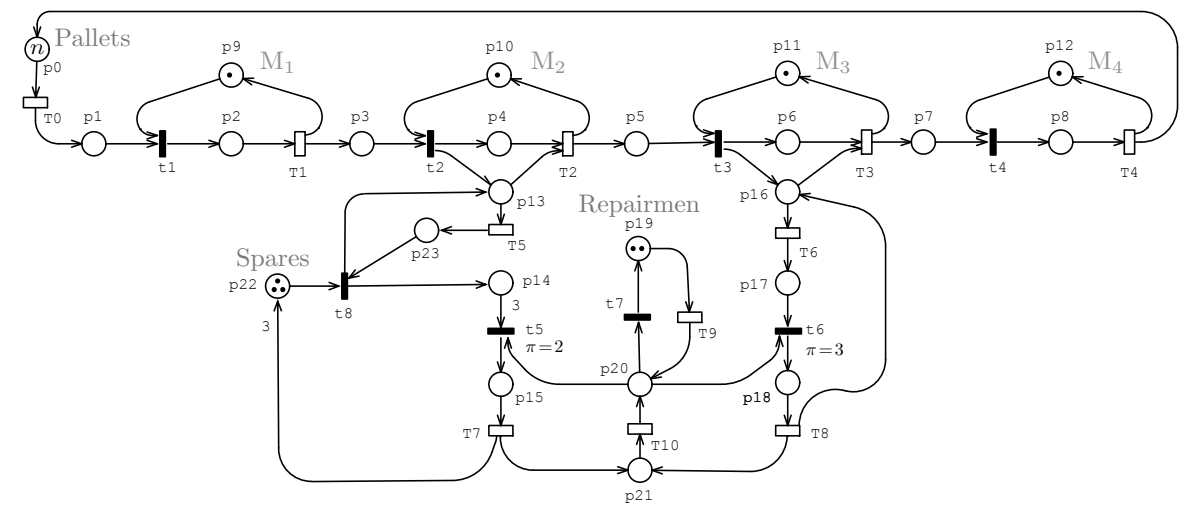

FIGURE 1. A FMS with faulting machines and repairmen.

in the model (repairmen, pallets, machines, spares). These entities have cyclic behaviors that repeat forever. Because of such a feature, which is specific of this model, in the sequel of this paper we often use the notion of circuits (set of places sequentially visited by tokens) that is useful to keep the explanations simple and intuitive, but that is not required for the results presented in this paper to hold.

\section{TAGGED TOKEN SPECIFICATION}

We already pointed out that in GSPN tokens are "volatile" elements created and destroyed on transition firings and with no semantic relationship between destroyed and created tokens. First passage times are defined at the level of the CTMC that underlies a GSPN, as measures of the time taken by the chain to move for the first time from a set of start states into a set of target states (target states are absorbing). Since start and target states correspond to specific distributions of tokens on the net places, it is natural to associate passage times to movements of certain tokens through the net that thus assume a distinctive identity meaningful for the modeler. Consider, for instance, the FMS model of Fig. 1. Tokens in places $p_{0}, p_{1}$, up to $p_{8}$ can be interpreted as the $N$ pallets that carry the parts from machine to machine in order to have them manufactured. Similarly, tokens in places $p_{15}, p_{18}, p_{19}$, $p_{20}$, and $p_{21}$ represent the repairmen in different states of their activities.

When the model is not trivial, identifying the groups of tokens that represent classes of trackable and atomic objects, as well as the parts of the model where they can flow through, is not a simple task: hence it is useful to give some support to the modeler in this function.

A rigorous notion of trackable objects can be provided looking for conservative flows of tokens in the net.

This approach not only supports the identification of the classes of objects suited for a first passage time analysis, but also contributes to the correctness of the results, since it is the basis for defining both a class of customers that behave similarly and the subnet where these customers flow.

The formal method that we use for this purpose is the computation of the minimal p-semiflows of the GSPN; to illustrate this concept let us refer again to the GSPN of Fig. 1. Default (automatically generated) names have been used to identify the places of this net to stress the conceptual separation between the mathematical properties derived from an automatic tool and their interpretation by the modeler.

The net has twelve minimal p-semiflows, illustrated in Tab. 1, related to different classes of objects. Most of them can be directly and easily interpreted by the modeler on the basis of his/her knowledge of the functionalities of this FMS application:

- $\mathbf{f}_{1}=\mathrm{p} 0+\mathrm{p} 1+\mathrm{p} 2+\mathrm{p} 3+\mathrm{p} 4+\mathrm{p} 5+\mathrm{p} 6+\mathrm{p} 7+\mathrm{p} 8$ : identifies the circuit followed by the pallets serving the different machines and the load-unload station;

- The p-semiflows $\mathbf{f}_{2}, \mathbf{f}_{3}$, and $\mathbf{f}_{4}$

$\mathbf{f}_{3}=\mathrm{p} 0+\mathrm{p} 1+\mathrm{p} 2+\mathrm{p} 3+\mathrm{p} 13+\mathrm{p} 23+\mathrm{p} 5+\mathrm{p} 6+\mathrm{p} 7+\mathrm{p} 8$

$\mathbf{f}_{4}=\mathrm{p} 0+\mathrm{p} 1+\mathrm{p} 2+\mathrm{p} 3+\mathrm{p} 4+\mathrm{p} 5+\mathrm{p} 16+\mathrm{p} 17+\mathrm{p} 18+\mathrm{p} 7+\mathrm{p} 8$

$\mathbf{f}_{2}=\mathrm{p} 0+\mathrm{p} 1+\mathrm{p} 2+\mathrm{p} 3+\mathrm{p} 13+\mathrm{p} 23+\mathrm{p} 5+\mathrm{p} 16+\mathrm{p} 17+\mathrm{p} 18+\mathrm{p} 7+\mathrm{p} 8$

also identify the circuit followed by the pallets, but put in evidence the fact that when a pallet is processed by machine $M 2$ or by machine $M 3$, its processing may be interleaved with periods of unavailability of the machine, due to a breakdown. Hence these can be interpreted as more refined representations of the pallets circuit.

- $\mathbf{f}_{5}=\mathrm{p} 2+\mathrm{p} 9, \mathbf{f}_{6}=\mathrm{p} 4+\mathrm{p} 10, \mathbf{f}_{7}=\mathrm{p} 6+\mathrm{p} 11, \mathbf{f}_{8}=\mathrm{p} 8+\mathrm{p} 12$ : these p-semiflows are related to the manufacturing machines $\mathrm{M}_{1}$ through $\mathrm{M}_{4}$ and state that each individual machine can be either busy (places p2, p4, p6, p8 marked), or idle (places p9, p10, p11, p12 marked);

- $\mathbf{f}_{10}=\mathrm{p} 11+\mathrm{p} 16+\mathrm{p} 17+\mathrm{p} 18$ : this $\mathrm{p}$-semiflow is related to machine $\mathrm{M}_{3}$ and states that it may be either idle (place p11 marked), busy (place p16 marked), waiting for a repairman (place p17 marked), or being repaired (place p18 marked);

- $\mathbf{f}_{9}=\mathrm{p} 10+\mathrm{p} 13+\mathrm{p} 23$ : this $\mathrm{p}$-semiflow is related to machine $\mathrm{M}_{2}$ and states that it may be either idle (place p10 marked), busy (place p13 marked), or waiting for a spare (place p23 marked); 
TABLE 1. Minimal p-semiflows of the FMS GSPN model. In bold the subnet $\mathcal{N}_{\mathbf{f}}$ of p-semiflow $\mathbf{f}$.

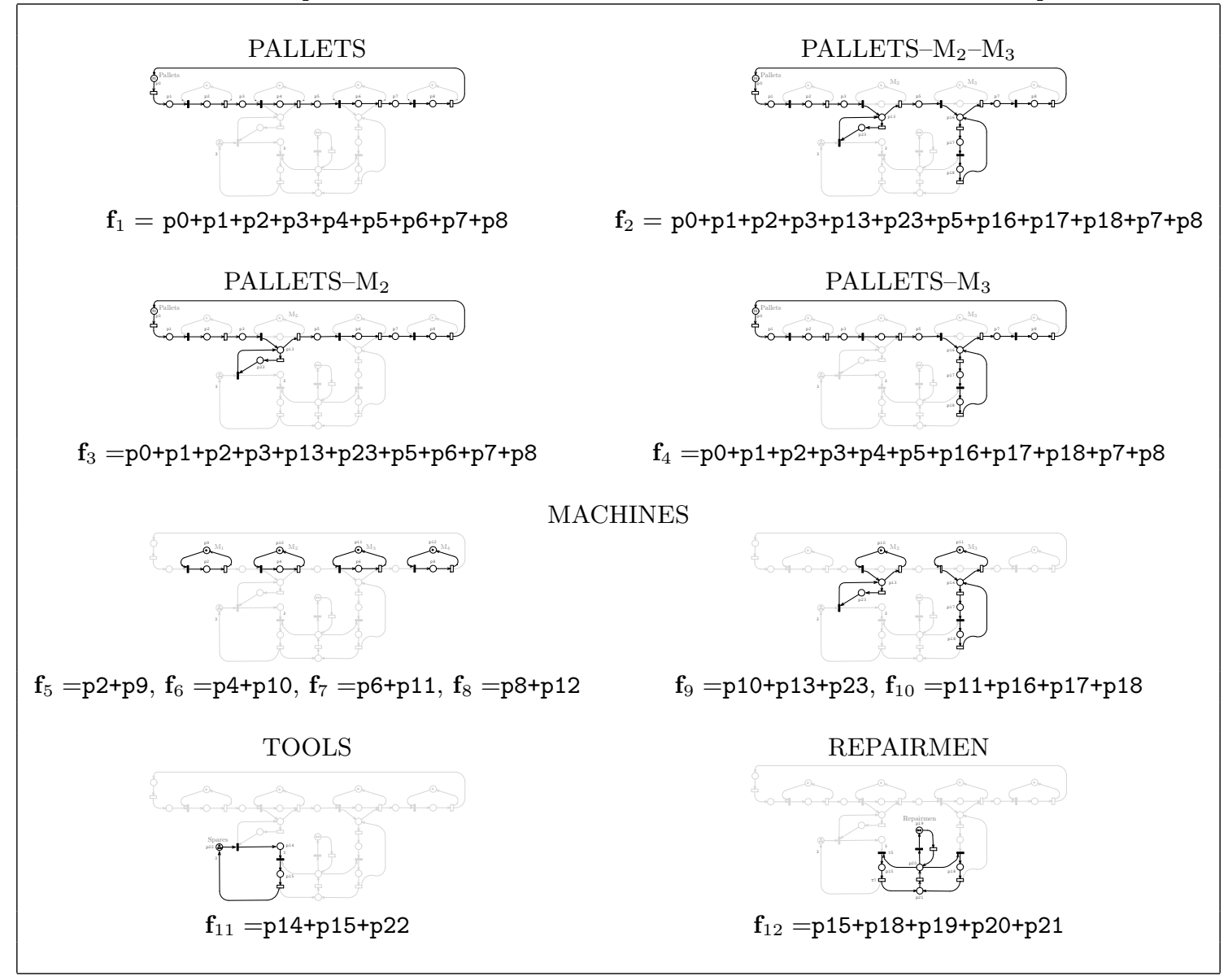

- $\mathbf{f}_{12}=\mathrm{p} 19+\mathrm{p} 20+\mathrm{p} 21+\mathrm{p} 15+\mathrm{p} 18$ : this p-semiflow identifies the repairman class, it states that a repairman can be either in vacation (place p19 marked), in service (place p20 marked), working (places p15 or p18 marked), or resting after a repair (place p21 marked);

- $\mathbf{f}_{11}=\mathrm{p} 14+\mathrm{p} 15+\mathrm{p} 22$ : identifies the subnet of the spares, and states that a spare tool can be available (place p22 marked), just used and faulty (place p14 marked), or being repaired (place 14 marked);

With these interpretations, we can choose one of these p-semiflows to identify the entity for which we want to compute the first passage times of interest. For instance, we could assess the efficiency of the system from the point of view of the average length of the intervals between repairman vacations, or of the probability that the processing-time taken by the pallets to go through the portion of the system that may fail (machines $M_{2}$ and $M_{3}$ ) remains below a certain threshold.

The distribution of the first passage time is computed for the submodel of interest by means of a tagging procedure which refines the subnet in order to single out the interaction of one of the tokens of a certain class w.r.t. the others. Starting from a GSPN annotated with the indication of the p-semiflow of interest, a new (unfolded) GSPN can be built which accounts for the identity of one of these tokens and explicitly encodes it into the net structure. The identified customer is denoted as the tagged-customer, while the related token, that carries its identity in the whole net, the taggedtoken. The extended net captures the dynamics of the tagged customer w.r.t. the others and can thus answer the performance questions of interest.

The whole tagging procedure can be summarized with the following steps: (a) the minimal p-semiflows of the model are computed and proposed to the modeler as eligible classes of customers; (b) the modeler, on the basis of his/her knowledge of the application and of the goals of the analysis, selects one of the semiflows, thus identifying the class of customers of interest; (c) the subnet corresponding to the chosen semiflow is modified in order to track one token out of the group for first passage time analysis (see Sec. 5). The subnet modification done in this last step of the procedure, might require additional information on the characteristics of the model such as the scheduling discipline used to manage the tokens of a tagged place. By default a Random Order ( $\mathrm{RO}$ ) policy is adopted, however several other policies may be specified, as will be discussed in Sec. 6, that are irrelevant for the computation of average performance indices (of 
untagged models), but that become important for the correct computation of the first passage time distribution.

The formal definition of Tagged GSPN (TGSPN) follows:

Definition 3.1. Tagged GSPN: A Tagged GSPN, $T G S P N$, is a tuple $\left(\mathcal{N}, \mathbf{f}, p_{\circ}, \mathcal{Q}, n\right)$ such that:

1. $\mathcal{N}=\left(P, T, I^{-}, I^{+}, H, \Pi, \mathbf{w}, \mathbf{m}_{0}\right)$ is a $G S P N$;

2. $\mathbf{f}$ is a minimal p-semiflow of the GSPN. It identifies the sets $\mathcal{P} \subseteq P$ and $\mathcal{T} \subseteq T$ such that $\mathcal{P}=\left\{p_{i} \in \mathbf{f}\right\}$, $\mathcal{T}=\left\{t_{j}: p_{i} \in \mathcal{P} \wedge\left(t_{j} \in \bullet p_{i} \vee t_{j} \in p_{i}^{\bullet}\right) \wedge I^{-}\left(t_{j}, p_{i}\right)-\right.$ $\left.I^{+}\left(t_{j}, p_{i}\right) \neq 0\right\}$

3. $\forall t \in \mathcal{T}:\left|t^{\bullet} \cap \mathcal{P}\right|=1$, meaning that a tugged transition cannot have more than one tagged output place.

4. $p_{\circ}$ is the place where the tagged-token is located in the initial marking $\mathbf{m}_{0}$ and is chosen so that $p_{\circ} \in \mathcal{P}$ : $\mathbf{m}_{0}\left(p_{\circ}\right)>0$;

5. The places in $\mathcal{P}$ can be interpreted as queues that may contain both tagged and untagged tokens: this distinction makes it important to specify a scheduling policy for such queues; the default scheduling policy is Random Order (RO). $\mathcal{Q}$ maps each queue place to one of the following scheduling policies: Random Order (RO), First Come First Served (FCFS), Last Come First Served with No Preemption (LCFS), Last Come First Served with Preemption-Resume (LCFS$P R)$; if the policy of place $p_{\circ}$ is not $R O$, then the initial position $1 \leqslant n \leqslant \mathbf{m}_{0}\left(p_{\circ}\right)$ of the token in the queue must be specified.

6. Some restrictions are imposed ${ }^{3}$ on each place $p \in \mathcal{P}$ with a scheduling policy different from $R O$ :

(i) $p$ can have at most one tagged transition $t$ in its output set;

(ii) if $t$ is timed, it can be of type $n$-server with a given rate $\lambda$, or have a marking dependent rate (which is interpreted as a marking dependent rate of a single server). $t$ cannot be of type infinite server.

(iii) if $t$ is immediate, it can have a constant or a marking dependent weight.

The third point of Def. 3.1 (requiring no more than one output tagged place for each tagged transition) is set to minimize the amount of additional information required for the model to be unambiguously specified: in fact if a tagged transition had more than one tagged output place, the modeler should provide (possibly probabilistic, or marking dependent) criteria to decide where the tagged token should be added when the transition fires extracting the tagged toked from one input place. Despite the restrictions, Def. 3.1 of TGSPN is not less general than that in [2], on the contrary, it enhances the model with the possibility to specify scheduling policies different from RO.

\footnotetext{
${ }^{3}$ These restrictions are mainly introduced to keep the subsequent formal development simpler: in the next sections it will be discussed how they can be relaxed.
}

\section{NET DEFINITION OF FIRST PASSAGE TIME}

One of the most challenging points of this problem is to provide a clear and easy-to-use net level specification of passage times that enables the modeler to define the measures of interest on the net rather than on its underlying CTMC. The passage of tokens representing customers throughout the net corresponds to traversal paths that they follow and along which timing intervals are measured. Focusing the attention on the class of customers identified by a p-semiflow $\mathbf{f}$, we can identify the subnet $\mathcal{N}_{\mathbf{f}}$ that is a restriction of $\mathcal{N}$ on the sets of places and transitions respectively identified by $\mathcal{P}$ and $\mathcal{T}$, given in Def. 3.1 of TGSPN. Referring to the FMS running example, Table 1 highlights the subnets that can be identified for this model.

Passage time information is provided identifying, within $\mathcal{N}_{\mathbf{f}}$, a set of open paths. In its simplest form, a path is identified by entry and exit points; however forbidden exit points along the route could also be specified. The definition of entry and exit points is based on the notion of events that cause the tagged customer to enter, and subsequently leave, the path (or set of paths) of interest. This is obtained by identifying specific transitions of $\mathcal{N}_{\mathbf{f}}$ that we call entry and exit points, denoted with sets $\mathcal{T}_{\text {in }}$ and $\mathcal{T}_{\text {out }}$

The connected component of $\mathcal{N}_{\mathbf{f}}$ that is defined following the oriented arcs from the entry to the exit/forbidden-points, embeds all the traversal-paths along which to compute the passage time. It is a subnet $\mathcal{N}_{\mathcal{S}}$ identified by set $\mathcal{S}$ of nodes (places and transitions).

Forbidden paths are specified through forbidden exit-points (denoted by the set $\mathcal{T}_{\text {forb }}$ ). These forbidden transitions allow to identify a subset of paths starting with a transition in $\mathcal{T}_{\text {in }}$ that should not be considered when computing the passage time distribution. Forbidden exit points translate into forbidden (absorbing) states of the CTMC used for first passage time computation [13].

Notice, that the existence of forbidden states makes the passage time distribution of interest "defective" [13]. The set of places and transitions $\mathcal{S}$ corresponding to the traversal subnet $\mathcal{N}_{\mathcal{S}}$ is derived as follows: $\mathcal{S}=\mathcal{S}^{\prime} \cup \mathcal{T}_{\text {in }} \cup \mathcal{T}_{\text {out }} \cup \mathcal{T}_{\text {forb }}$.

Having defined ${ }^{4} \mathcal{T}_{i n}^{\bullet}$ as the union of all postset of the transitions in $\mathcal{T}_{i n}, \mathcal{S}^{\prime}$ is the smallest set of nodes (places and transitions) that satisfy the following conditions:

- $\mathcal{T}_{i n}^{\bullet} \subseteq \mathcal{S}^{\prime}$

- if $n \in \mathcal{S}^{\prime}$ then $n^{\prime} \in n^{\bullet} \backslash\left(\mathcal{T}_{\text {out }} \cup \mathcal{T}_{\text {forb }}\right)$ is also in $\mathcal{S}^{\prime}$

- $\mathcal{N}_{\mathcal{S}}$ is the subnet with all nodes in $\mathcal{S}$ and all arcs of $\mathcal{N}_{\mathrm{f}}$ connecting them.

In order for the traversal subnet to be consistent, it is required that $\mathcal{S}^{\prime} \cap \mathcal{T}_{i n}=\emptyset$; this condition means

\footnotetext{
${ }^{4}$ It is important to remark that the $t^{\bullet}$ in this context is the output set of $t$ in $\mathcal{N}_{\mathbf{f}}$.
} 


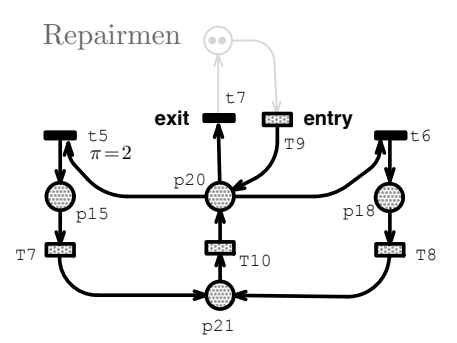

FIGURE 2. Traversal subnet for the repairmen's measure (in bold).

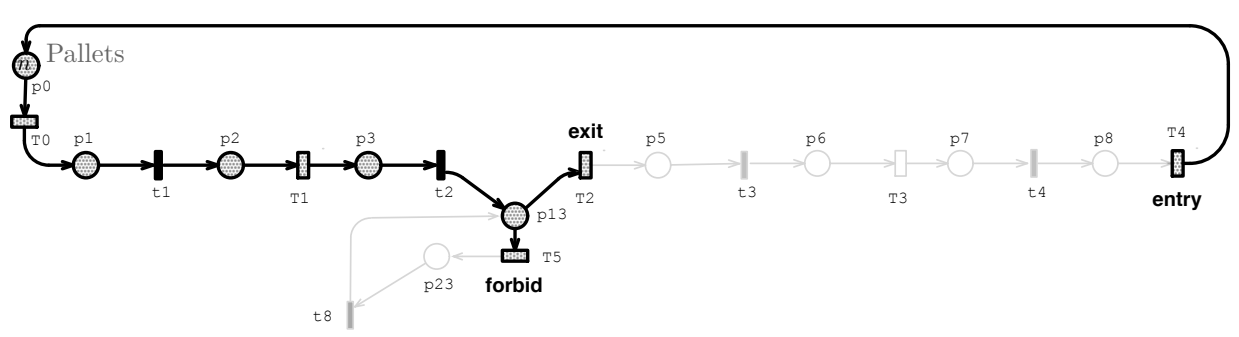

FIGURE 3. Traversal subnet for the pallets' measure (in bold). that there cannot be traversal paths in $\mathcal{S}$ that allow to reach an entry point from any entry point, without encountering an exit or forbidden point along the way.

The sets $\mathcal{T}_{\text {in }}, \mathcal{T}_{\text {out }}$, and $\mathcal{T}_{\text {forb }}$ allow a straightforward construction of the sets of start, target, and forbidden states that may be refined by means of boolean expressions on the local markings of places (e.g., one may be interested in the time spent in a given subnet by the tagged customer, conditioned on the fact that no other customer was in the subnet upon its arrival). Given a traversal subnet $\mathcal{N}_{\mathcal{S}}$ consistent with the above definition, start, end, and forbidden states are characterized by corresponding sets of triplets ENTRY $/ E X I T / F O R B I D=\left\{\left\langle t, C_{t}^{\text {pre }}, C_{t}^{\text {post }}\right\rangle\right\}$ one for each element of the sets of entry, exit, forbidden points (respectively) $t \in \mathcal{T}_{\text {in }} / t \in \mathcal{T}_{\text {out }} / t \in$ $\mathcal{T}_{\text {forb }} . \quad C_{t}^{\text {pre }}, C_{t}^{\text {post }}$ are boolean conditions on the (tangible) marking preceding/following the firing of the corresponding transition. These conditions must be satisfied by start,end, or forbidden states $\left(C_{t}^{\text {post }}\right)$ and by their predecessors $\left(C_{t}^{\text {pre }}\right)$ in the (tangible) $\mathrm{RG}$, and are used to restrict the measure of the first passage time to some specific conditions (of course the conditions can be defined as true if no restrictions are applied).

For instance, in the FMS model of Fig. 1, considering the activities of the repairmen, the modeler can decide to specify the computation of the first passage time from the beginning of a working cycle (firing of transition T9) up to the end of that cycle (firing of transitions t7). However if he is interested only in the definition of those repair cycles in which some repair has to be performed, he must add the following condition on the marking of places p14 and p17: either place p14 or place p17 must contain enough tokens to require a repair service start, before the firing of T9 $(E N T R Y=\{\langle\mathrm{T} 9, \mathbf{m}(\mathrm{p} 14)>3 \vee$ $\mathbf{m}(\mathrm{p} 17)>0$, true $\rangle\}, E X I T=\{\langle\mathrm{t} 7$, true, true $\rangle\})$. The resulting subnet is illustrated in Fig. 2, where T9 is the entry-point and $t 7$ is the exit-point. An equivalent specification of the start and end states directly through the description of the markings would require the presence of the tagged token either in p15 or in p18 for the start states, and in place p19 for the end states.

Another example, in this case concerning the possible paths of the pallets, is the specification of the time required to pass through a given sequence of machines, for example for loading a new part on the pallet (including the time spent in queue), and then to process it with M1 and M2 in sequence. In this case the entry point would be T4, and the exit point T2. If the modeler is interested in computing the passage time distribution for this sequence, but only for those cases in which machine M2 does not break down while processing the tagged customer, it is possible to specify a forbidden exit point, namely $\mathrm{T} 5$, possibly with the additional condition that the tagged token be in p23 in the tangible marking reached after its firing (observe that in order to have the possibility of specifying such forbidden exit point either $\mathbf{f}_{2}$ or $\mathbf{f}_{3}$ must be chosen as the minimal semiflow describing the customer's path; the additional condition on p23 is useful to avoid considering as forbidden a route that has no impact on the service time, due to the immediate return of the tagged token in p13 after the firing of T5 immediately followed by t8). The resulting subnet is illustrated in Fig. 3.

A state based specification of the same measure should indicate the presence of the tagged token in p0 for characterizing start states, and the presence of the tagged token in place p5 or p6 for characterizing end states. Forbidden states could be characterized by the (tangible) presence of the tagged token in p23.

Note that the TGSPN formalism allows to associate different scheduling policies with places: in formalisms without this feature any scheduling policy different from Random Order should be explicitly modeled. If in the running example an FCFS scheduling policy is declared for p0, the previous entry point definition of the passage time measure remains valid. On the other hand in a model where $\mathrm{p} 0$ is explicitly substituted with a FCFS queue submodel the state based specification of the same measure should be updated to account for the presence of the tagged token in any place representing a specific position in the queue. In general, whenever the entry point transition firing is followed by a sequence of immediate steps that may lead the tagged token in different final positions, a state based specification is more error prone. A similar problem arises when traversal paths may involve cycles within the subnet of interest: let us consider for example the model of a machine with service time distributed as an Erlang-k and with possible failures (upon their repairs 


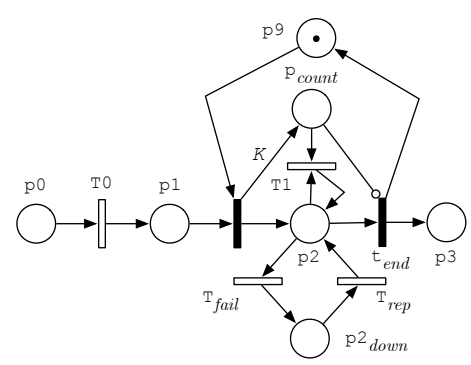

FIGURE 4. An example of a machine with Erlang-K.

the processing restarts from the point where it was interrupted), as depicted in Fig. 4: if one is interested in the distribution of the time needed to get through the machine, it is possible to simply specify entry point T0 and exit point $t_{e n d}$. A state based definition of the start states should require the presence of tagged token in $\mathrm{p} 1$, or the presence of tagged token in $\mathrm{p} 2$ provided that $\mathbf{m}\left(\mathrm{p}_{\text {count }}\right)=K$ : this specification must be carefully handled in the passage time computation process, as discussed in details in Sec. 7. The end state would simply be defined as tagged token in p3 (and possibly other places if the token can immediately move forward from $\mathrm{p} 3$ to other places).

Observe that the current definition does not allow the computation of cycle time distributions since this would require the possibility of considering a transition as both an entry and exit point simultaneously, however this extension does not pose conceptual problems: it can be managed at the level of the CTMC by replicating the start states to break the cyclic paths from start state to start state, and make the replicated state, at the end of the path, absorbing.

\section{TGSPN SEMANTICS AND UNFOLDING}

In this section we define the semantics of a TGSPN in terms of a GSPN constructed following an unfolding procedure. The following diagram shows the relation among the models discussed in this section:

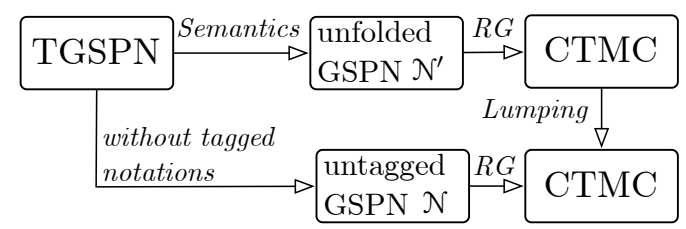

by unfolding the TGSPN we define its semantics, since the behavior of a TGSPN corresponds to the behavior of the GSPN $\mathcal{N}^{\prime}$ derived from it through unfolding. If instead we ignore the tagging annotations of a TGSPN we obtain another GSPN $\mathcal{N}$, whose behavior is an abstraction of $\mathcal{N}^{\prime}$ (and hence of the TGSPN). It is possible to establish a relation between the reachable states of $\mathcal{N}$ and $\mathcal{N}^{\prime}$, in particular each state in $\mathcal{N}^{\prime}$ is a refinement of one state in $\mathcal{N}$. We denote $R S(\mathcal{N})$ the reachability set of net $\mathcal{N}$ and with $R S\left(\mathcal{N}^{\prime}\right)$ the reachability set of net $\mathcal{N}^{\prime}$. In the last part of this section we will prove that the CTMC derived from $\mathcal{N}^{\prime}$ is strongly lumpable, when we consider aggregates of refined states corresponding to the same abstract state, and its lumping produces a CTMC isomorphic to that of $\mathcal{N}$.

As we already pointed out, p-semiflow $\mathbf{f}$ in the definition of the TGSPN identifies a subnet which is related to a particular class of customers (or tokens): this subnet and the tokens marking its places are thus the objects of the tagging procedure. The unfolding amounts to isolating one of these customers and to explicitly representing the ways in which it interacts with the others. This corresponds to duplicating the subnet followed by the tokens so that one copy is reserved to the tagged token while the other copy is reserved to the other tokens of the class.

To formalize this intuitive explanation, given a $\operatorname{TGSPN}\left(\mathcal{N}, \mathbf{f}, p_{\circ}, \mathcal{Q}, n\right)$, let us define the subnet $\mathcal{N}_{\mathbf{f}}^{+}$as the subgraph of $\mathcal{N}$ defined by the restriction of $\mathcal{N}$ on the set of transitions $\mathcal{T}$ and on the set of places $\bigcup_{t \in \mathcal{T}}\left(\bullet t \cup t^{\bullet}\right)$. In this section we assume that a RO policy is implicitly adopted for all the queue places of the net. In Sec. 6 we will discuss how to deal with other policies.

To better illustrate the unfolding procedure, Fig. 5 is provided as a reference: it depicts the process for a single tagged transition (with only one input tagged place). Part (a) depicts a TGSPN fragment, where transition $t_{i}$, and places $p_{j}$ and $p_{l}$ are tagged, i.e., $t_{i} \in \mathcal{T}$ and $p_{j}, p_{l} \in \mathcal{P}$. Part (b) presents the unfolded GSPN: transition $t_{i, j}^{\mathrm{t}}$, and places $p_{j}^{\mathrm{t}}$ and $p_{l}^{\mathrm{t}}$ constitute part of the unfolding of $t_{i}, p_{j}$, and $p_{l}$, related to the flow of the tagged token. Transition $t_{i}^{\mathrm{u}}$, and places $p_{j}^{\mathrm{u}}$ and $p_{l}^{\mathrm{u}}$ instead is the part of the unfolding of $t_{i}, p_{j}$, and $p_{l}$ related to the flow of the untagged tokens.

Let $\left(\mathcal{N}, \mathbf{f}, p_{\circ}, \mathcal{Q}\right)$ be a TGSPN, where

$$
\mathcal{N}=\left(T, P, I^{-}, I^{+}, H, \Pi, \mathbf{w}, \mathbf{m}_{0}\right)
$$

its unfolding is the GSPN

$$
\mathcal{N}^{\prime}=\left(T^{\prime}, P^{\prime}, I^{\prime-}, I^{\prime+}, H^{\prime}, \Pi^{\prime}, \mathbf{w}^{\prime}, \mathbf{m}_{0}^{\prime}\right)
$$

defined as follows.

Let $\mathcal{T}^{\prime}, \mathcal{P}^{\prime}$ and $P^{+}$be sets defined in the following manner: $\mathcal{P}^{\prime}=\left\{p_{i}^{\mathrm{t}}, p_{i}^{\mathrm{u}}: p_{i} \in \mathcal{P}\right\} . \quad \mathcal{P}^{\prime}$ represents the refinement of $\mathcal{P}$ : For each place $p_{i}$ in $\mathcal{P}$ of the TGSPN,

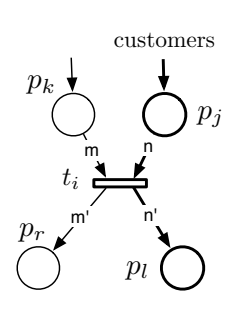

(a)

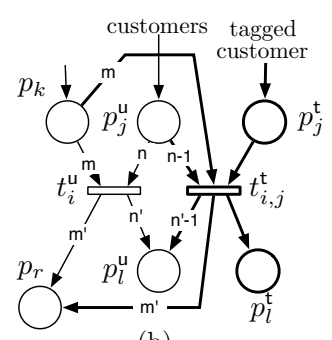

(b)
FIGURE 5. Unfolding procedure (a) TGSPN; (b) unfolded GSPN. 
there exists in the GSPN one untagged place $p_{i}^{\mathrm{u}}$ which will contain all tokens, but the tagged one, and one tagged place $p_{i}^{\mathrm{t}}$ which will contain the tagged token.

$\mathcal{T}^{\prime}=\left\{t_{i, j}^{\mathrm{t}}, t_{i}^{\mathrm{u}}: t_{i} \in \mathcal{T} \wedge p_{j} \in{ }^{\bullet} t_{i} \cap \mathcal{P}\right\}$. $\mathcal{T}^{\prime}$ represents the refinement of $\mathcal{T}$. For each transition $t_{i}$ in $\mathcal{T}$ of the TGSPN, there exists in the GSPN one untagged transition $t_{i}^{\mathrm{u}}$ which will fire for all tokens, but the tagged one, and one or more tagged transitions $t_{i, j}^{\mathrm{t}}$ which will fire for the tagged token in tagged place $p_{j}^{\mathrm{t}}$.

$P^{+}=\left\{p_{i} \in \mathcal{P}: \exists t_{j}\right.$ such that $H\left(t_{j}, p_{i}\right) \neq \infty \mathrm{V}$ $\left.I^{-}\left(t_{j}, p_{i}\right)=I^{+}\left(t_{j}, p_{i}\right)\right\}$. Set $P^{+}$is a set of additional places: they are used in the unfolding to translate tagged places in $\mathcal{P}$ which act like either inhibitors or test conditions. Then $\mathcal{N}^{\prime}$ is defined as follows, where to avoid useless repetitions, $I^{*}$ stands for both $I^{-}$and $I^{+}$:

- $T^{\prime}=T \backslash \mathcal{T} \cup \mathcal{T}^{\prime}$

- $P^{\prime}=P \backslash \mathcal{P} \cup \mathcal{P}^{\prime} \cup P^{+}$

- $\forall t_{i}^{\mathrm{u}} \in \mathcal{T}^{\prime}, p_{j}^{\mathrm{u}} \in \mathcal{P}^{\prime}, p_{j}^{\mathrm{t}} \in \mathcal{P}^{\prime}, p_{k} \in P \backslash \mathcal{P}$ :

$I^{\prime *}\left(t_{i}^{\mathrm{u}}, p_{j}^{\mathrm{u}}\right)=I^{*}\left(t_{i}, p_{j}\right), I^{\prime *}\left(t_{i}^{\mathrm{u}}, p_{k}\right)=I^{*}\left(t, p_{k}\right)$,

$I^{\prime *}\left(t_{i}^{\mathrm{u}}, p_{j}^{\mathrm{t}}\right)=0$

- $\forall t_{i, j}^{\mathrm{t}} \in \mathcal{T}^{\prime}$

- if $p_{k} \in P \backslash \mathcal{P}$ then $I^{*}\left(t_{i, j}^{\mathrm{t}}, p_{k}\right)=I^{*}\left(t_{i}, p_{k}\right)$

- if $p_{k} \in{ }^{\bullet} t_{i} \cap \mathcal{P} \wedge I^{-}\left(t_{i}, p_{k}\right) \neq I^{+}\left(t_{i}, p_{k}\right)$

$I^{\prime-}\left(t_{i, j}^{\mathrm{t}}, p_{k}^{\mathrm{t}}\right)= \begin{cases}1 & k=j \\ 0 & k \neq j\end{cases}$

$I^{\prime-}\left(t_{i, j}^{\mathrm{t}}, p_{k}^{\mathrm{u}}\right)= \begin{cases}I^{-}\left(t_{i}, p_{k}\right)-1 & k=j \\ I^{-}\left(t_{i}, p_{k}\right) & k \neq j\end{cases}$

otherwise ${I^{\prime}}^{-}\left(t_{i, j}^{\mathrm{t}}, p_{k}^{\mathrm{t}}\right)={I^{\prime}}^{-}\left(t_{i, j}^{\mathrm{t}}, p_{k}^{\mathrm{u}}\right)=0$

- if $p_{k} \in t_{i}^{\bullet} \cap \mathcal{P} \wedge I^{-}\left(t_{i}, p_{k}\right) \neq I^{+}\left(t_{i}, p_{k}\right)$

$I^{\prime+}\left(t_{i, j}^{\mathrm{t}}, p_{k}^{\mathrm{u}}\right)=I^{+}\left(t_{i}, p_{k}\right)-1$

$I^{\prime+}\left(t_{i, j}^{\mathrm{t}}, p_{k}^{\mathrm{t}}\right)=1$

otherwise $I^{\prime+}\left(t_{i, j}^{\mathrm{t}}, p_{k}^{\mathrm{t}}\right)=I^{\prime+}\left(t_{i, j}^{\mathrm{t}}, p_{k}^{\mathrm{u}}\right)=0$

- $\forall t_{i} \in T \backslash \mathcal{T}$ and $\forall p_{j} \in P \backslash \mathcal{P}$

$I^{\prime *}\left(t_{i}, p_{j}\right)=I^{*}\left(t_{i}, p_{j}\right)$ and $H^{\prime}\left(t_{i}, p_{j}\right)=H\left(t_{i}, p_{j}\right)$

- $\forall p_{i} \in P^{+}:{ }^{\bullet} p_{i}=\bullet p_{i}^{\mathrm{u}} \cup \bullet p_{i}^{\mathrm{t}}$ and $p_{i}^{\bullet}=p_{i}^{\mathrm{u}} \bullet p_{i}^{\mathrm{t}} \bullet$, if $t_{k} \in T \backslash \mathcal{T}$ then $H^{\prime}\left(t_{k}, p_{i}\right)=H\left(t_{k}, p_{i}\right), I^{\prime *}\left(t_{k}, p_{i}\right)=$ $I^{*}\left(t_{k}, p_{i}\right)$;

if $t_{k} \in \mathcal{T}$ then $H^{\prime}\left(t_{k, j}^{\mathrm{t}}, p_{i}\right)=H^{\prime}\left(t_{k, j}^{\mathrm{u}}, p_{i}\right)=H\left(t_{k}, p_{i}\right)$, $I^{* *}\left(t_{k, j}^{\mathrm{t}}, p_{i}\right)=I^{* *}\left(t_{k, j}^{\mathrm{u}, j}, p_{i}\right)=I^{*}\left(t_{k}, p_{i}\right), \quad \forall j$

The initial marking $\mathbf{m}_{0}^{\prime}$ of $\mathcal{N}^{\prime}$ is:

- $\forall p_{i} \in \mathcal{P}: p_{i} \neq p_{\circ}$ then $\mathbf{m}_{0}^{\prime}\left(p_{i}^{\mathrm{u}}\right)=\mathbf{m}_{0}\left(p_{i}\right) \wedge$ $\mathbf{m}_{0}^{\prime}\left(p_{i}^{\mathrm{t}}\right)=0$

- $\mathbf{m}_{0}^{\prime}\left(p_{\circ}^{\mathrm{u}}\right)=\mathbf{m}_{0}\left(p_{\circ}\right)-1 \wedge \mathbf{m}_{0}^{\prime}\left(p_{\circ}^{\mathrm{t}}\right)=1$

- $\forall p_{i} \in P \backslash \mathcal{P} \cup P^{+} \mathbf{m}_{0}^{\prime}\left(p_{i}\right)=\mathbf{m}_{0}\left(p_{i}\right)$

The firing weights and rates $\mathbf{w}^{\prime}$ of $\mathcal{N}^{\prime}$ are defined as follows. Observe that the formulas below are valid for timed transitions whose input arc from a tagged place has multiplicity one. A general formula for the case of arc multiplicity greater than one, is provided at the end of the section. The two following expressions recur often in the next paragraphs, and are denoted:

$$
\mathrm{w}^{\mathrm{t}}=\frac{\mathbf{m}\left(p_{j}^{\mathrm{u}}\right)}{\mathbf{m}\left(p_{j}^{\mathrm{u}}\right)+\mathbf{m}\left(p_{j}^{\mathrm{t}}\right)} ; \quad \mathrm{w}^{\mathrm{u}}=\frac{\mathbf{m}\left(p_{j}^{\mathrm{t}}\right)}{\mathbf{m}\left(p_{j}^{\mathrm{u}}\right)+\mathbf{m}\left(p_{j}^{\mathrm{t}}\right)}
$$

they represent respectively the probability that the tagged copy or the untagged copy of a given transition fire (assuming that the only input tagged place of the transition is $p_{j}$ ): when one of these two expressions evaluate to zero, the corresponding transition copy is not enabled in the unfolded net.

Immediate transitions: if transition $t_{i} \in \mathcal{T}$ is an immediate transition with weight $w\left(t_{i}\right)$, then its unfolded copies $t_{i, j}^{\mathrm{t}} \in T^{\prime}$ and $t_{i}^{\mathrm{u}} \in T^{\prime}$ in the new GSPN remain immediate with marking dependent weight:

$$
w^{\prime}\left(t_{i}^{\mathrm{u}}, \mathbf{m}\right)=w\left(t_{i}\right) \mathrm{w}^{\mathrm{u}} ; \quad w^{\prime}\left(t_{i, j}^{\mathrm{t}}, \mathbf{m}\right)=w\left(t_{i}\right) \mathrm{w}^{\mathrm{t}}
$$

These formulae consider the fact that transitions $t_{i}^{\mathrm{u}}$ and $t_{i, j}^{\mathrm{t}}$ may be conflicting (due to sharing of input places) and solve the conflict giving to each copy a probability of firing proportional to the number of customer-tokens involved in the conflict. If the net contains chains of causally connected immediate transitions leading to more complex situations of indirect conflict [12], then it may be necessary to explicitly ask additional information to the modeler.

Timed transitions of type Infinite Server: if transition $t_{i} \in \mathcal{T}$ is a timed transition of type "Infinite Server" and rate $w\left(t_{i}\right)$, then its unfolded copies $t_{i}^{\mathrm{t}} \in T^{\prime}$ and $t_{i}^{\mathrm{u}} \in T^{\prime}$ in the GSPN remain timed of type "Infinite Server" with rates $w^{\prime}\left(t_{i}^{\mathrm{u}}\right)=w\left(t_{i, j}^{\mathrm{t}}\right)=w\left(t_{i}\right)$;

Timed transitions of type n-Server: if transition $t_{i} \in \mathcal{T}$ is a timed transition of type "n-Server" and rate $w\left(t_{i}\right)$, then its unfolded copies $t_{i}^{\mathrm{t}} \in T^{\prime}$ and $t_{i}^{\mathrm{u}} \in T^{\prime}$ in the new GSPN remain timed, but of type "Marking Dependent" with rates (the $*$ stands for either $\mathrm{t}$ or $\mathrm{u}$ ):

$$
w^{\prime}\left(t_{i}^{*}, \mathbf{m}\right)=\min \left\{\mathbf{m}\left(p_{j}^{\mathrm{u}}\right)+\mathbf{m}\left(p_{j}^{\mathrm{t}}\right), n\right\} w\left(t_{i}\right) \mathrm{w}^{*}
$$

The above expressions are valid only if in the TGSPN $p_{j}$ is the only place in the input set of $t_{i}$. In a more general situation, $e d\left(t_{i}, \mathbf{m}\right)$ must be considered in place of $\min \left\{\mathbf{m}\left(p_{j}^{\mathrm{u}}\right)+\mathbf{m}\left(p_{j}^{\mathrm{t}}\right), n\right\}$.

Timed transitions of type Marking Dependent: if transition $t_{i} \in \mathcal{T}$ is a timed transition of type "Marking Dependent", assume that the marking dependent rate of transition $t_{i}$ is expressed as a function of the marking, i.e., $g(\mathbf{m})$. Let $p_{j} \in \mathcal{P}$ the tagged place input of $t_{i}$. Then the unfolded copies of $t_{i}, t_{i}^{\mathrm{t}} \in T^{\prime}$ and $t_{i}^{\mathrm{u}} \in T^{\prime}$, in the new GSPN remain timed of type "Marking Dependent" with rates that are modified in the following manner: $g^{*}$, the marking dependent function of transition $t_{i, j}^{*}$, is obtained by $g$ after an appropriate variables substitution for any value of $k$ such that $p_{k} \in \mathcal{P}$ :

$$
g^{*}(\mathbf{m})=\mathbf{w}^{*} \cdot g\left(\mathbf{m}\left(p_{1}\right), \ldots, \mathbf{m}\left(p_{k}^{\mathrm{u}}\right)+\mathbf{m}\left(p_{k}^{\mathrm{t}}\right), \ldots, \mathbf{m}\left(p_{n}\right)\right)
$$

Let us now consider the more complex case of a tagged place connected to a transition by means of 
TABLE 2. Several subnets $\mathcal{N}_{\mathrm{f}}^{+}$related to minimal psemiflows for the FMS GSPN model.

\begin{tabular}{cc|} 
PALLETS \\
$\mathcal{N}_{\mathbf{f}_{1}}^{+}$ & REPAIRMEN \\
$\mathcal{N}_{\mathbf{f}_{12}}^{+}$ \\
\end{tabular}

an arc of multiplicity $n$ greater than one. When $t_{i}$ fires, $n$ tokens are withdrawn from $p$ : the probability that the tagged token is included among the $n$ tokens selected by the firing of transition $t_{i}$, is $n / \mathbf{m}(p)$. If the transition has $\mathrm{k}$-servers or infinite servers, the same idea can be applied, since in any case we consider only one transition firing at a time: the only thing that changes in this case is that the firing rate of such transitions will be $\mathbf{w}\left(t_{i}, \mathbf{m}\right) * e d\left(t_{i}, \mathbf{m}\right)$.

As a consequence the formulas above have to be updated by substituting with:

$$
\begin{gathered}
\mathrm{w}^{\mathrm{t}}=\frac{n \mathbf{m}\left(p_{j}^{\mathrm{t}}\right)}{\mathbf{m}\left(p_{j}^{\mathrm{u}}\right)+\mathbf{m}\left(p_{j}^{\mathrm{t}}\right)} \\
\mathrm{w}^{\mathrm{u}}=\frac{\mathbf{m}\left(p_{j}^{\mathrm{u}}\right)-(n-1) \mathbf{m}\left(p_{j}^{\mathrm{t}}\right)}{\mathbf{m}\left(p_{j}^{\mathrm{u}}\right)+\mathbf{m}\left(p_{j}^{\mathrm{t}}\right)}
\end{gathered}
$$

Finally, in case there are several tagged input places $p_{1}, \ldots, p_{m}$ connected to a given transition $t$ through arcs of multiplicity $k_{1}, \ldots, k_{m}$ respectively, then the term corresponding to $t^{\mathrm{u}}$ must be modified as follows:

$$
\mathrm{w}^{\mathrm{u}}=\prod_{i=1, m} \frac{\mathbf{m}\left(p_{i}^{\mathrm{u}}\right)-\left(k_{i}-1\right) \mathbf{m}\left(p_{i}^{\mathrm{t}}\right)}{\mathbf{m}\left(p_{i}^{\mathrm{u}}\right)+\mathbf{m}\left(p_{i}^{\mathrm{t}}\right)}
$$

The terms of the product will either be all equal to one (in case no tagged token is present in any input place) or all but one will be equal to one (in case one of the tagged places contains the tagged token: by construction at most one of them can contain such token).

\subsection{Examples of unfolding}

This section illustrates several net unfoldings for the FMS model with regard to different classes of possible customers. Table 2 highlights two of the subnets that may be involved in the unfolding step according to which class of customers is considered by the modeler as the target for a first passage time analysis. Subnet $\mathcal{N}_{\mathbf{f}_{1}}^{+}$regards the pallets, subnet $\mathcal{N}_{\mathbf{f}_{12}}^{+}$refers to the repairmen. Notice these subnets are the $\mathcal{N}_{\mathbf{f}_{*}}$ augmented with additional places that do not pertain to the flow of customers, but that contain items that influence the transition enabling and firing.
Focusing on the behavior of the pallets, Fig. 6 illustrates the unfolding for their first passage time analysis. The portion of the representation resulting from the unfolding of $\mathcal{N}_{\mathbf{f}_{1}}^{+}$is darker, whereas the untouched part in the final GSPN is lighter. The block on the bottom right corner corresponds to the modification of the net needed to implement a FCFS policy that will be discussed in details in Sec. 6

More complex situations than those arising in our running example are possible and need to be addressed in order to show the generality of our unfolding procedure. Consider the case depicted in Fig. 7(a) where the three places of the net are covered by a minimal p-semiflow $\left(p_{1}+p_{2}+p_{3}\right)$ and where two tagged places are input to the same transition $t_{1}$.

The tokens moving over the tagged places of this example must join (synchronize) in order for transition $t_{1}$ to fire, the tagged token can join with a companion token either coming from place $p_{1}$ or place $p_{3}$. Hence $t_{1}$ unfolds into $t_{1}^{\mathrm{u}}, t_{1,1}^{\mathrm{t}}$, and $t_{1,3}^{\mathrm{t}}$. Another aspect illustrated in the example is the translation of transitions with arc's multiplicity greater than 1 .

\subsection{Models equivalence}

The unfolding procedure generates a new model whose behavior can be interpreted as a refinement of the behavior of the GSPN derived from the TGSPN eliminating all the annotations. Indeed it is possible to define a mapping from states $\mathbf{m}_{i} \in R S\left(\mathcal{N}^{\prime}\right)$ of the unfolded net to states $\widehat{\mathbf{m}} \in R S(\mathcal{N})$ of the more abstract GSPN, as detailed hereafter.

Let us rename the places so that places of $\mathbf{f}$ are lexicographically before the other ones. Let us represent $\widehat{\mathbf{m}} \in R S(\mathcal{N})$ explicitly by means of a vector indexed on the place names:

$$
[\underbrace{\widehat{\mathbf{m}}\left(p_{1}\right), \widehat{\mathbf{m}}\left(p_{2}\right), \ldots, \widehat{\mathbf{m}}\left(p_{n}\right)}_{\mathcal{P}}, \underbrace{\widehat{\mathbf{m}}\left(p_{n+1}\right), \ldots, \widehat{\mathbf{m}}\left(p_{m}\right)}_{P \backslash \mathcal{P}}]
$$

We say that a marking $\mathbf{m}_{i}$ in $R S\left(\mathcal{N}^{\prime}\right)$ belongs to aggregate $\widehat{\mathbf{m}}$, denoted $\mathcal{A}\left(\mathbf{m}_{i}\right)=\widehat{\mathbf{m}}$, iff it is so defined:

$$
\begin{aligned}
& {[\underbrace{\mathbf{m}_{i}\left(p_{1}^{\mathrm{t}}\right), \mathbf{m}_{i}\left(p_{1}^{\mathrm{u}}\right), \mathbf{m}_{i}\left(p_{2}^{\mathrm{t}}\right), \mathbf{m}_{i}\left(p_{2}^{\mathrm{u}}\right), \ldots, \mathbf{m}_{i}\left(p_{n}^{\mathrm{t}}\right), \mathbf{m}_{i}\left(p_{n}^{\mathrm{u}}\right)}_{P \backslash \mathcal{P}},} \\
& \underbrace{\mathbf{m}_{i}\left(p_{n+1}\right), \ldots, \mathbf{m}_{i}\left(p_{m}\right)}_{\mathcal{P}^{\prime}}]
\end{aligned}
$$

where $\mathbf{m}_{i}\left(p_{k}^{\mathrm{t}}\right)+\mathbf{m}_{i}\left(p_{k}^{\mathrm{u}}\right)=\widehat{\mathbf{m}}\left(p_{k}\right), \forall p_{k} \in \mathcal{P}$, and $\mathbf{m}_{i}\left(p_{k}\right)=$ $\widehat{\mathbf{m}}\left(p_{k}\right), \forall p_{k} \in P \backslash \mathcal{P}$.

Actually, the refined marking contains also the places in $P^{+}$, used only in case of inhibitor or test arcs from tagged places. Their marking is equal to the marking of the corresponding place in the abstract state. We omit these places from the discussion to avoid confusion, but the extension to consider them is straightforward because places in $P^{+}$can be treated as places in $P \backslash \mathcal{P}$.

There is a relation between the p-semiflows of $\mathcal{N}$ and those of $\mathcal{N}^{\prime}$. In fact, the p-semiflow $\mathbf{f}$ identifying the 


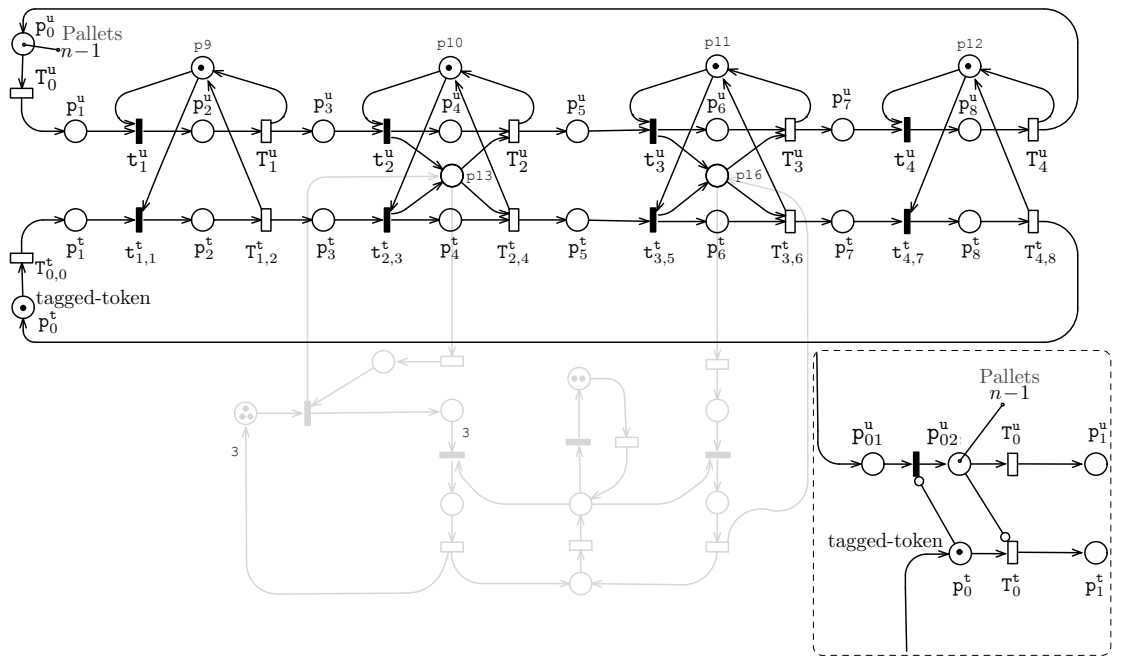

FIGURE 6. Unfolded GSPN for the pallets class of customers, $\mathcal{N}_{\mathbf{f}_{1}}^{+}$, and its variation when p0 has a FCFS scheduling policy.

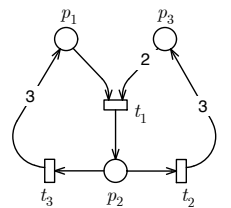

(a)

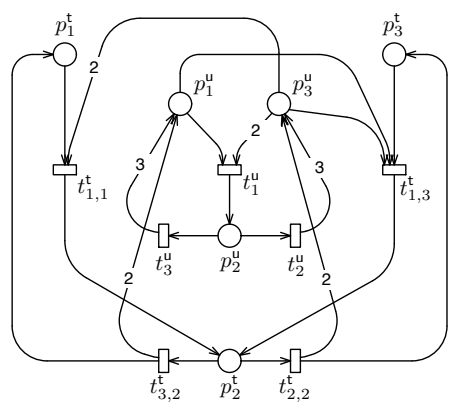

(b)

FIGURE 7. Unfolded GSPN. subnet subject to unfolding, is split into two p-semiflows in the unfolded net $\mathcal{N}^{\prime}$ : one comprising the places $p_{i}^{\mathrm{t}}$ whose marking represents the position of the tagged token, and one comprising their untagged counterpart $p_{i}^{\mathrm{u}}$. Denoting $\mathbf{f}^{\mathrm{t}}$ and $\mathbf{f}^{\mathrm{u}}$ these $\mathrm{p}$-semiflows, assuming that $\mathbf{f} \cdot \widehat{\mathbf{m}}_{0}=K$ in $\mathcal{N}$, the following invariants hold $\forall \mathbf{m} \in R S\left(\mathcal{N}^{\prime}\right):$

$\mathbf{f}^{\mathrm{t}} \cdot \mathbf{m}=\mathbf{f}^{\mathrm{t}} \cdot \mathbf{m}_{0}=1$ and $\mathbf{f}^{\mathrm{u}} \cdot \mathbf{m}=\mathbf{f}^{\mathrm{u}} \cdot \mathbf{m}_{0}=K-1$

From the invariants above we can conclude that $\mathbf{m}_{i}\left(p_{k}^{\mathrm{t}}\right) \in\{0,1\}$, moreover one and only one place $p_{k}^{\mathrm{t}}$ has one token. Hence, each abstract state $\widehat{\mathbf{m}}$ is associated with at most $n$ refined states. Subscript $i$ in $\mathbf{m}_{i}$ denotes the place index where the tagged token is located that is, $\mathcal{A}\left(\mathbf{m}_{i}\right)=\widehat{\mathbf{m}}$ and $\mathbf{m}_{i}\left(p_{i}^{\mathrm{u}}\right)=\widehat{\mathbf{m}}\left(p_{i}\right)-1 \wedge \mathbf{m}_{i}\left(p_{i}^{\mathrm{t}}\right)=1$.

To simplify the notation, in the rest of this section we denote $t_{i}^{\mathrm{t}}$, with single subscript $i$, the tagged unfolding of transition $t$ connected to place $p_{i}^{\mathrm{t}}$ (i.e. we omit the transition subscript when not needed). Figure 8 is used as reference to illustrate the relationships between $R S(\mathcal{N})$ and $R S\left(\mathcal{N}^{\prime}\right)$ in the two following Lemma.

Lemma 5.1. Let $\mathcal{A}\left(\mathbf{m}_{i}\right)=\widehat{\mathbf{m}}$ and $\mathcal{A}\left(\mathbf{m}_{i}^{\prime}\right)=\widehat{\mathbf{m}}^{\prime}$.

i. $\forall t \in T \backslash \mathcal{T}$,

$$
t \in \mathcal{E}(\widehat{\mathbf{m}}) \wedge \widehat{\mathbf{m}}[t\rangle \widehat{\mathbf{m}}^{\prime} \Longleftrightarrow t \in \mathcal{E}\left(\mathbf{m}_{i}\right) \wedge \mathbf{m}_{i}[t\rangle \mathbf{m}_{i}^{\prime}
$$

ii. $\forall t \in \mathcal{T}$,

$$
\begin{aligned}
& t \in \mathcal{E}(\widehat{\mathbf{m}}) \wedge \widehat{\mathbf{m}}[t\rangle \widehat{\mathbf{m}}^{\prime} \Longleftrightarrow \\
& \text { one among the two situations happens: }
\end{aligned}
$$

1. $t_{i}^{t} \in \mathcal{E}\left(\mathbf{m}_{i}\right) \wedge t^{u} \notin \mathcal{E}\left(\mathbf{m}_{i}\right)$ and $\mathbf{m}_{i}\left[t_{i}^{t}\right\rangle \mathbf{m}_{j}^{\prime}$

2. $t_{i}^{t} \in \mathcal{E}\left(\mathbf{m}_{i}\right) \wedge t^{u} \in \mathcal{E}\left(\mathbf{m}_{i}\right)$ and $\mathbf{m}_{i}\left[t^{u}\right\rangle \mathbf{m}_{i}^{\prime} \wedge \mathbf{m}_{i}\left[t_{i}^{t}\right\rangle \mathbf{m}_{j}^{\prime}$

Moreover if $\forall k t_{k}^{t} \notin \mathcal{E}\left(\mathbf{m}_{i}\right) \wedge p_{i} \notin \bullet$ then

3. $t \in \mathcal{E}(\widehat{\mathbf{m}}) \wedge \widehat{\mathbf{m}}[t\rangle \widehat{\mathbf{m}}^{\prime} \Longleftrightarrow t^{u} \in \mathcal{E}\left(\mathbf{m}_{i}\right) \wedge \mathbf{m}_{i}\left[t^{u}\right\rangle \mathbf{m}_{i}^{\prime}$
Proof. Case $i$. Referring to diagram of Fig. 8 case $i$. accounts for state transition labeled (3). If $t \in \mathcal{E}(\widehat{\mathbf{m}})$ then $t \in \mathcal{E}\left(\mathbf{m}_{i}\right) \forall i$ and vice versa, in fact the input places of $t$ are not tagged, hence they have the same marking in $\widehat{\mathbf{m}}$ and $\mathbf{m}_{i}$. Now, by construction (see unfolding rules (4)) $p_{j} \in{ }^{\bullet} t$ or $p_{j} \in t^{\bullet}$ iff $p_{j} \in P \backslash \mathcal{P}$. In $R S(\mathcal{N})$ marking $\widehat{\mathbf{m}}^{\prime}\left(p_{j}\right)$ is:

$$
\widehat{\mathbf{m}}^{\prime}\left(p_{j}\right)=\widehat{\mathbf{m}}\left(p_{j}\right)-I^{-}\left(t, p_{j}\right)+I^{+}\left(t, p_{j}\right)
$$

respectively in $R S\left(\mathcal{N}^{\prime}\right)$ marking $\mathbf{m}_{i}^{\prime}\left(p_{j}\right)$ is:

$$
\mathbf{m}_{i}^{\prime}\left(p_{j}\right)=\mathbf{m}_{i}\left(p_{j}\right)-I^{\prime-}\left(t, p_{j}\right)+I^{\prime+}\left(t, p_{j}\right)
$$

applying the unfolding rules in (4) and the definition of aggregates it is:

$$
\begin{aligned}
& \mathbf{m}_{i}^{\prime}\left(p_{j}\right)=\widehat{\mathbf{m}}\left(p_{j}\right)-I^{-}\left(t, p_{j}\right)+I^{+}\left(t, p_{j}\right) \\
& \mathbf{m}_{i}^{\prime}\left(p_{j}\right)=\widehat{\mathbf{m}}^{\prime}\left(p_{j}\right)
\end{aligned}
$$

Since the above is valid for all places $p_{j}$ which are input or output of $t$ then $\mathbf{m}_{i}^{\prime}$, such that $\mathbf{m}_{i}[t\rangle \mathbf{m}_{i}^{\prime}$, is a refinement of $\widehat{\mathbf{m}}^{\prime}$.

\section{Case $i$.}

Let us first prove the common part of $i i .1$ and ii.2, that is, if $\widehat{\mathbf{m}}[t\rangle \widehat{\mathbf{m}}^{\prime}$ then $\mathbf{m}_{i}\left[t_{i}^{t}\right\rangle \mathbf{m}_{j}^{\prime}$ and vice versa. Referring to diagram of Fig. 8, we are in the case depicted by the state transitions labeled with (1). Let us show that the enabling conditions for each place belonging to $t_{i}^{\mathrm{t}}$ are verified iff $t \in \mathcal{E}(\widehat{\mathbf{m}})$.

If $p_{k} \in P \backslash \mathcal{P}$ then $\mathbf{m}_{i}\left(p_{k}\right)=\widehat{\mathbf{m}}\left(p_{k}\right)$ by definition of aggregates. Moreover $I^{\prime-}\left(t_{j}^{\mathrm{t}}, p_{k}\right)=I^{-}\left(t, p_{k}\right) \forall j$ and $\mathbf{m}_{i}\left(p_{k}\right) \geq I^{\prime-}\left(t_{j}^{\mathrm{t}}, p_{k}\right), \forall j$ iff $\widehat{\mathbf{m}}\left(p_{k}\right) \geq I^{-}\left(t, p_{k}\right)$.

If $p_{i} \in \mathcal{P}$. By definition of aggregate, $\widehat{\mathbf{m}}\left(p_{i}\right) \geq I^{-}\left(t, p_{i}\right)$ iff $\mathbf{m}_{i}\left(p_{i}^{\mathrm{t}}\right)=1$ and $\mathbf{m}_{i}\left(p_{i}^{\mathrm{u}}\right)=\widehat{\mathbf{m}}\left(p_{i}\right)-1$ (we recall that in marking $\mathbf{m}_{i}$ the tagged token is in place $\left.p_{i}^{\mathrm{t}}\right)$. Now, $\widehat{\mathbf{m}}\left(p_{i}\right) \geq I\left(t, p_{i}\right)$ iff $\widehat{\mathbf{m}}\left(p_{i}\right)-1 \geq I\left(t, p_{i}\right)-1$. The first term of inequality is equal to $\mathbf{m}_{i}\left(p_{i}^{\mathrm{u}}\right)$ and the second is equal to $I^{\prime-}\left(t_{i}^{\mathrm{t}}, p_{i}^{\mathrm{u}}\right)$ (unfolding rule $(1)$ ). That is $\widehat{\mathbf{m}}\left(p_{i}\right) \geq I^{-}\left(t, p_{i}\right)$ iff $\mathbf{m}_{i}\left(p_{i}^{\mathrm{u}}\right) \geq I^{\prime-}\left(t_{i}^{\mathrm{t}}, p_{i}^{\mathrm{u}}\right)$. For what concern the tagged place it is $\mathbf{m}_{i}\left(p_{i}^{\mathrm{t}}\right)=1=I^{\prime-}\left(t_{i}^{\mathrm{t}}, p_{i}^{\mathrm{t}}\right)$. If $p_{j \neq i} \in(\bullet t \cap \mathcal{P})$. For the tagged version $p_{j}^{\mathrm{t}}$ it 
is $I^{\prime-}\left(t_{i}^{\mathrm{t}}, p_{j}^{\mathrm{t}}\right)=0$; for the untagged version $p_{j}^{\mathrm{u}}$ it is $\mathbf{m}_{i}\left(p_{j}^{\mathrm{u}}\right)=\widehat{\mathbf{m}}\left(p_{j}\right)$ (aggregate definition) and $I^{\prime-}\left(t_{i}^{\mathrm{t}}, p_{j}^{\mathrm{u}}\right)=$ $I^{-}\left(t_{i}, p_{j}\right)$ (unfolding rule (1)). Hence, $\mathbf{m}_{i}\left(p_{j}^{\mathrm{u}}\right) \geq I^{\prime-}\left(t_{i}^{\mathrm{t}}, p_{j}^{\mathrm{u}}\right)$ iff $\widehat{\mathbf{m}}\left(p_{j}\right) \geq I^{-}\left(t_{i}, p_{j}\right)$.

The above proves that $t_{i}^{\mathrm{t}} \in \mathcal{E}\left(\mathbf{m}_{i}\right)$ iff $t \in \mathcal{E}(\widehat{\mathbf{m}})$. Let us prove the second part, that is, $t_{i}^{\mathrm{t}}$ firing in $\mathbf{m}_{i}$ leads to a marking which is a refinement of $\widehat{\mathbf{m}}^{\prime}$, and vice versa. We have two kind of places $p_{j} \in P \backslash \mathcal{P}$ and $p_{j} \in \mathcal{P}$.

If $p_{j} \in \mathcal{P}$. It holds:

$$
\begin{aligned}
& \mathbf{m}_{x}^{\prime}\left(p_{j}^{\mathrm{u}}\right)=\mathbf{m}_{i}\left(p_{j}^{\mathrm{u}}\right)-I^{\prime-}\left(t_{i}^{\mathrm{t}}, p_{j}^{\mathrm{u}}\right)+I^{\prime+}\left(t_{i}^{\mathrm{t}}, p_{j}^{\mathrm{u}}\right) \\
& \mathbf{m}_{x}^{\prime}\left(p_{j}^{\mathrm{t}}\right)=\mathbf{m}_{i}\left(p_{j}^{\mathrm{t}}\right)-I^{\prime-}\left(t_{i}^{\mathrm{t}}, p_{j}^{\mathrm{t}}\right)+I^{\prime+}\left(t_{i}^{\mathrm{t}}, p_{j}^{\mathrm{t}}\right)
\end{aligned}
$$

Summing member-wise the above we obtain:

$$
\mathbf{m}_{x}^{\prime}\left(p_{j}^{\mathrm{u}}\right)+\mathbf{m}_{x}^{\prime}\left(p_{j}^{\mathrm{t}}\right)=\mathbf{m}_{i}\left(p_{j}^{\mathrm{u}}\right)+\mathbf{m}_{i}\left(p_{j}^{\mathrm{t}}\right)-I^{\prime-}\left(t_{i}^{\mathrm{t}}, p_{j}^{\mathrm{u}}\right)-
$$$$
I^{\prime-}\left(t_{i}^{\mathrm{t}}, p_{j}^{\mathrm{t}}\right)+I^{\prime+}\left(t_{i}^{\mathrm{t}}, p_{j}^{\mathrm{u}}\right)+I^{\prime+}\left(t_{i}^{\mathrm{t}}, p_{j}^{\mathrm{t}}\right)
$$

If $j=i$ and $p_{i} \in \boldsymbol{\bullet}_{i}$ substituting using unfolding rules

(2)(branches $k=j$ ):

$$
\begin{aligned}
& \mathbf{m}_{x}^{\prime}\left(p_{i}^{\mathbf{u}}\right)+\mathbf{m}_{x}^{\prime}\left(p_{i}^{\mathrm{t}}\right)=\widehat{\mathbf{m}}\left(p_{i}\right)-\left[I^{-}\left(t_{i}, p_{i}\right)-1+1\right] \\
& \mathbf{m}_{x}^{\prime}\left(p_{i}^{\mathrm{u}}\right)+\mathbf{m}_{x}^{\prime}\left(p_{i}^{\mathrm{t}}\right)=\widehat{\mathbf{m}}\left(p_{i}\right)-I^{-}\left(t_{i}, p_{i}\right) \\
& \mathbf{m}_{x}^{\prime}\left(p_{i}^{\mathrm{u}}\right)+\mathbf{m}_{x}^{\prime}\left(p_{i}^{\mathrm{t}}\right)=\widehat{\mathbf{m}}^{\prime}\left(p_{i}\right)
\end{aligned}
$$

If $j \neq i$ and $p_{j} \in t_{i}$ substituting using unfolding rules

(2) (branches for $k \neq j$ ):

$$
\begin{aligned}
& \mathbf{m}_{x}^{\prime}\left(p_{j}^{\mathrm{u}}\right)+\mathbf{m}_{x}^{\prime}\left(p_{j}^{\mathrm{t}}\right)=\widehat{\mathbf{m}}\left(p_{j}\right)-I^{-}\left(t_{i}, p_{j}\right) \\
& \mathbf{m}_{x}^{\prime}\left(p_{j}^{\mathrm{u}}\right)+\mathbf{m}_{x}^{\prime}\left(p_{j}^{\mathrm{t}}\right)=\widehat{\mathbf{m}}^{\prime}\left(p_{j}\right)
\end{aligned}
$$

If $p_{j} \in t_{i}^{\bullet}$ substituting using unfolding rules (3):

$\mathbf{m}_{x}^{\prime}\left(p_{j}^{\mathbf{u}}\right)+\mathbf{m}_{x}^{\prime}\left(p_{j}^{\mathbf{t}}\right)=\widehat{\mathbf{m}}\left(p_{j}\right)+\left[I^{+}\left(t_{i}, p_{j}\right)-1+1\right]$

$\mathbf{m}_{x}^{\prime}\left(p_{j}^{\mathrm{u}}\right)+\mathbf{m}_{x}^{\prime}\left(p_{j}^{\mathrm{t}}\right)=\widehat{\mathbf{m}}\left(p_{j}\right)+I^{+}\left(t_{i}, p_{j}\right)$

$\mathbf{m}_{x}^{\prime}\left(p_{j}^{\mathrm{u}}\right)+\mathbf{m}_{x}^{\prime}\left(p_{j}^{\mathrm{t}}\right)=\widehat{\mathbf{m}}^{\prime}\left(p_{j}\right)$

If $p_{j} \in P \backslash \mathcal{P}$

$$
\mathbf{m}_{x}^{\prime}\left(p_{j}\right)=\mathbf{m}_{i}\left(p_{j}\right)-I^{\prime-}\left(t_{i}^{\mathrm{t}}, p_{j}\right)+I^{\prime+}\left(t_{i}^{\mathrm{t}}, p_{j}\right)
$$

due to unfolding rules (1) and due to definition of aggregates:

$$
\begin{aligned}
& \mathbf{m}_{x}^{\prime}\left(p_{j}\right)=\widehat{\widehat{\mathbf{m}}}\left(p_{j}\right)-I^{-}\left(t_{i}, p_{j}\right)+I^{+}\left(t_{i}, p_{j}\right) \\
& \mathbf{m}_{x}^{\prime}\left(p_{j}\right)=\widehat{\mathbf{m}}^{\prime}\left(p_{j}\right)
\end{aligned}
$$

Let us now prove that, assuming $t^{\mathrm{u}} \in \mathcal{E}\left(\widehat{\mathbf{m}}_{k}\right), \widehat{\mathbf{m}}[t\rangle \widehat{\mathbf{m}}^{\prime}$ iff $\mathbf{m}_{k}\left[t^{\mathrm{u}}\right\rangle \mathbf{m}_{k}^{\prime} . t$ firing in $\widehat{\mathbf{m}}$ modifies the marking of $p_{j}$ as:

$$
\widehat{\mathbf{m}}^{\prime}\left(p_{j}\right)=\widehat{\mathbf{m}}\left(p_{j}\right)-I^{-}\left(t, p_{j}\right)+I^{+}\left(t, p_{j}\right)
$$

Let us assume $p_{j} \in \mathcal{P}$. By unfolding rules in (1), $t^{\mathrm{u}}$ firing in $\mathbf{m}_{k}$ modifies $p_{j}^{\mathrm{u}}$ marking as:

$$
\begin{aligned}
& \mathbf{m}_{k}^{\prime}\left(p_{j}^{\mathrm{u}}\right)=\mathbf{m}_{k}\left(p_{j}^{\mathrm{u}}\right)-I^{-}\left(t, p_{j}\right)+I^{+}\left(t, p_{j}\right) \\
& \mathbf{m}_{k}^{\prime}\left(p_{j}^{\mathrm{t}}\right)=\mathbf{m}_{k}\left(p_{j}^{\mathrm{t}}\right)-0+0
\end{aligned}
$$

summing member-wise the above we obtain:

$$
\mathbf{m}_{k}^{\prime}\left(p_{j}^{\mathrm{u}}\right)+\mathbf{m}_{k}^{\prime}\left(p_{j}^{\mathrm{t}}\right)=\mathbf{m}_{i}\left(p_{j}^{\mathrm{u}}\right)+\mathbf{m}_{k}\left(p_{j}^{\mathrm{t}}\right)-I^{-}\left(t, p_{j}\right)+I^{+}\left(t, p_{j}\right)
$$

Since $\mathbf{m}_{k}$ is a refinement of $\widehat{\mathbf{m}}$, then it becomes:

$$
\mathbf{m}_{k}^{\prime}\left(p_{j}^{\mathrm{u}}\right)+\mathbf{m}_{k}^{\prime}\left(p_{j}^{\mathrm{t}}\right)=\widehat{\mathbf{m}}\left(p_{j}\right)-I^{-}\left(t, p_{j}\right)+I^{+}\left(t, p_{j}\right)
$$

and finally:

$$
\mathbf{m}_{k}^{\prime}\left(p_{j}^{\mathrm{u}}\right)+\mathbf{m}_{k}^{\prime}\left(p_{j}^{\mathbf{t}}\right)=\widehat{\mathbf{m}}^{\prime}\left(p_{j}\right)
$$

If $p_{j} \in P \backslash \mathcal{P}$ the proof is similar to the one provided for Case $i$. Hence, $t^{\mathrm{u}}$ firing leads to a marking of $R S\left(\mathcal{N}^{\prime}\right)$ which is a refinement of $\widehat{\mathbf{m}}^{\prime} \in R S\left(\mathcal{N}^{\prime}\right)$. The above derivation also says that if $t^{\mathrm{u}} \in \mathcal{E}\left(\mathbf{m}_{k}\right)$ and $\mathbf{m}_{k}\left[t^{\mathrm{u}}\right\rangle \mathbf{m}_{k}$ then $\widehat{\mathbf{m}}[t\rangle \widehat{\mathbf{m}}^{\prime}$. And thus we have completely proved $i i .2$.

The above proof holds for all $k$, in particular when $k=i$ it proves $i i .3$. Moreover the additional conditions $\forall t_{k}, t_{k}^{\mathrm{t}} \notin \mathcal{E}\left(\mathbf{m}_{i}\right) \wedge t \in \mathcal{E}(\widehat{\mathbf{m}})$ imply that $t^{\mathrm{u}} \in \mathcal{E}\left(\mathbf{m}_{i}\right)$. Referring to Fig. 8 , we are in the case depicted by transitions (2).

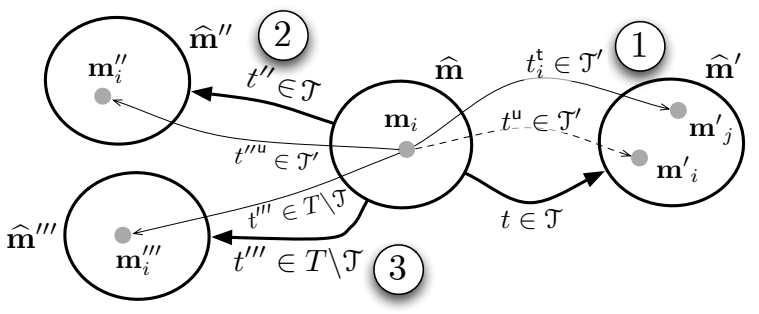

FIGURE 8. Aggregation relationships between $\mathbf{m}_{i}$ and $\widehat{\mathbf{m}}=\mathcal{A}\left(\mathbf{m}_{i}\right)$.

Lemma 5.2. Let $\mathbf{m} \in R S\left(\mathcal{N}^{\prime}\right)$, then

$$
\begin{aligned}
& \text { 1. } t_{i}^{t} \notin \mathcal{E}(\mathbf{m}) \wedge t^{u} \in \mathcal{E}(\mathbf{m}) \Longrightarrow w\left(t^{u}, \mathbf{m}\right)=w(t, \widehat{\mathbf{m}}) \\
& \text { 2. } t_{i}^{t} \in \mathcal{E}(\mathbf{m}) \wedge t^{u} \in \mathcal{E}(\mathbf{m}) \Longrightarrow w\left(t_{i}^{t}, \mathbf{m}\right)+w\left(t^{u}, \widehat{\mathbf{m}}\right)= \\
& w(t, \widehat{\mathbf{m}}) \\
& \text { 3. } t_{i}^{t} \in \mathcal{E}(\mathbf{m}) \wedge t^{u} \notin \mathcal{E}(\mathbf{m}) \Longrightarrow w\left(t_{i}^{t}, \mathbf{m}\right)=w(t, \widehat{\mathbf{m}})
\end{aligned}
$$

Proof. By construction (unfolding rules (4)) it holds that the weight or rate $w(t, \mathbf{m})$ of any unfolded transition $t \in T \backslash \mathcal{T}$ of the GSPN is equal to the weight of $t \in T \backslash \mathcal{T}$ of the TGSPN. It is straightforward to verify by the definition of aggregate that $w(t, \widehat{\mathbf{m}})=w\left(t, \mathbf{m}_{i}\right)$ $\forall \mathbf{m}_{i}: \mathcal{A}\left(\mathbf{m}_{i}\right)=\widehat{\mathbf{m}}$.

Let us now consider $t \in \mathcal{T}$ and analyze the relationships between $t$ and its unfolding $t_{i}^{\mathrm{t}}$ and $t^{\mathrm{u}}$. All places in $P^{+} \cup(P \backslash \mathcal{P})$ play the same role in the enabling of $t_{i}^{\mathrm{t}}$ and $t^{\mathrm{u}}$. Let us look at the other places.

In Sec. 5 we specified the rates, or weights, of unfolded transitions $t^{\mathrm{u}} \in \mathcal{T}^{\prime}$ and $t_{k}^{\mathrm{t}} \in \mathcal{T}^{\prime}$ as proportions of the weight $w(t, \mathbf{m})$ of $t \in \mathcal{T}$. Equations (5) and (6) provided the general proportional factors.

Next, we prove the three statements of the Lemma, that is, $t_{i}^{\mathrm{t}}$ and $t^{\mathrm{u}}$ will always fire with the same total probability, in case of immediate transitions, or with the same total rate, in case of timed transition:

1. Under the hypothesis of statement 1 . it holds:

$\mathbf{m}\left(p_{i}^{\mathrm{u}}\right) \geq I^{-}\left(t, p_{i}\right) \wedge \mathbf{m}\left(p_{i}^{\mathrm{t}}\right)=0$ otherwise $t_{i}^{\mathrm{t}} \in \mathcal{E}(\mathbf{m})$. Equations (5) and (6) respectively become:

$$
\frac{0}{\mathbf{m}\left(p_{i}^{\mathbf{u}}\right)+0}=0 \quad \text { and } \quad \frac{\mathbf{m}\left(p_{i}^{\mathbf{u}}\right)}{\mathbf{m}\left(p_{i}^{\mathbf{u}}\right)+0}=1
$$

2. Under the hypothesis of statement 2. it holds: $\mathbf{m}\left(p_{i}^{\mathrm{u}}\right) \geq I^{-}\left(t, p_{i}\right) \wedge \mathbf{m}\left(p_{i}^{\mathrm{t}}\right)=1$. Equations (5) and (6) resectively become:

$$
\frac{I^{-}\left(t, p_{i}\right)}{\mathbf{m}\left(p_{i}^{\mathrm{u}}\right)+1}
$$

and

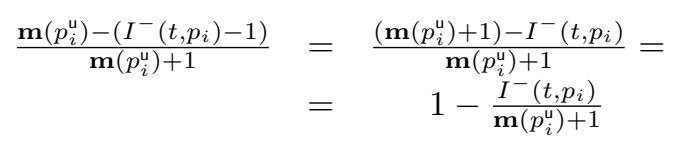

3. Under the hypothesis of statement 3. it holds: $\mathbf{m}\left(p_{i}^{\mathrm{u}}\right)=I^{-}\left(t, p_{i}\right)-1 \wedge \mathbf{m}\left(p_{i}^{\mathrm{t}}\right)=1$. Equations (5) and (6) respectively become: 


$$
\frac{I^{-}\left(t, p_{i}\right) \cdot 1}{I^{-}\left(t, p_{i}\right)-1+1}=1
$$

and

$$
\frac{\left(I^{-}\left(t, p_{i}\right)-1\right)-\left(I^{-}\left(t, p_{i}\right)-1\right)}{I^{-}\left(t, p_{i}\right)-1+1}=0
$$

As a consequence of Lemmas 5.1 and 5.2 , it is straightforward to show that the sum of weights of all transitions enabled in a marking $\widehat{\mathbf{m}}$ is equal to the sum of weights of all transitions enabled in any refinement $\mathbf{m}_{i}$ of $\widehat{\mathbf{m}}$. This ensures that all refinements of a tangible marking $\widehat{\mathbf{m}}$ have the same average sojourn time, moreover, considering vanishing markings, the statement of Lemma 5.2 holds true not only for transition weights but also for transition firing probabilities.

TheOREM 5.1. Given a TGSPN $\left(\mathcal{N}, p_{0}, \mathbf{f}, \mathcal{Q}\right)$, let $\mathcal{N}^{\prime}$ be the unfolded GSPN.

The embedded $M C$ derived from the $R G$ of $\mathcal{N}^{\prime}$ is lumpable w.r.t. the partition of the states that groups in a same aggregate the refined states corresponding to the same abstract state, moreover the resulting lumped embedded $M C$ is isomorphic to the embedded $M C$ obtained from the $R G$ of $\mathcal{N}$. The same holds for the CTMC derived from the TRG of the two nets.

Proof. Lemma 5.1 proves that it is possible to establish a correspondence between each transition $t$ firing in an abstract state $\widehat{\mathbf{m}}$ and groups of transition firings in any refinement $\mathbf{m}_{i}$ of $\widehat{\mathbf{m}}$ such that the latter lead to refinements in the same aggregate reached by $\widehat{\mathbf{m}}$ through $t$. Moreover the corresponding transitions have the same rate/probability, by Lemma 5.2. This implies that the strong lumpability condition holds, in fact the sum of the probabilities from a refined marking $\mathbf{m}_{i}$ to all refined markings in another aggregate $\widehat{\mathbf{m}}^{\prime}$, and the same sum from another refined marking $\mathbf{m}_{j}$, can both be expressed as sum of terms corresponding to the same transitions firing in aggregate $\widehat{\mathbf{m}}$.

\section{QUEUING POLICIES}

The definition of GSPNs does not explicitly address the way in which tokens in the input places of a transition are managed upon its firing. A random selection policy is commonly assumed which is not relevant when average measures are computed from these models.

When passage times are the quantities of interest and the tagged token approach is adopted, the policy used for the management of the tokens in the input places of a transition becomes, instead, relevant w.r.t. the results we want to compute. Up to now, all the results we have developed have been based on the standard definition of GSPN and thus on the default Random Order (RO) policy. In this section we relax

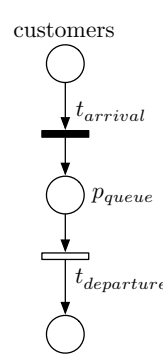

(a)

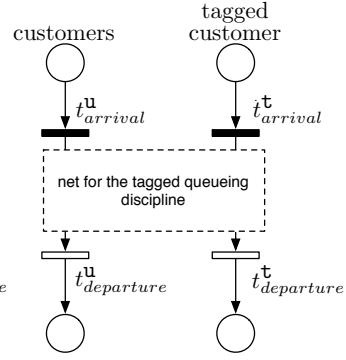

(b)
FIGURE 9. Untagged GSPN implicit queue.

this restriction and discuss several other policies that can be specified according to the TGSPN definition. In particular, the explicit representations of these policies using standard GSPN (that we call GSPN unfolding) are described at some length in order to derive efficient computational methods useful for handling these more complex cases.

According to the definition of TGSPN given in Sec. 3, tagged places are annotated with the policy used to manage their tokens; not annotated places (both tagged and untagged) are served in RO. When a place is annotated with a policy name, the TGSPN definition allows for a single transition to withdraw tokens from such a place. This restriction can be relaxed by allowing that same place to be part of the input set of several transitions as long as they are using the same policy and the multiplicities of their servers are identical. In this case we can show that there exists a transformation of this extended version of the net, which satisfies the restriction of the TGSPN definition and that is constructed using the general scheme depicted in Fig. 10.

In part (a) of such figure is the queuing place $p_{k}$ served by two $n$-server transitions $t_{i}$ and $t_{j}$ whose rates are $r_{i}$ and $r_{j}$, respectively. Because of the exponential distributions associated with the firings of transitions $t_{i}$ and $t_{j}$, this subnet is equivalent to that depicted in part (b) of the same figure where a single timed transition $t_{i, j}$ is connected to the queueing place and the direction actually followed by the token (which transition actually fired) is chosen after its firing via the competition between the two immediate transitions $t_{i}$ and $t_{j}$ characterized by the weights shown in the figure.

When a queue-place is input of an immediate transition, that transition is assumed to be a single server and the transformation is performed as in the timed transition case.

Currently we assume that queue places are managed according to the following (scheduling or queueing) disciplines: FCFS (First Come First Served), LCFS (Last Come First Served without preemption), LCFSPR (Last Come First Served with Preemption), besides the RO policy mentioned before.

When developing the GSPN representation of a 


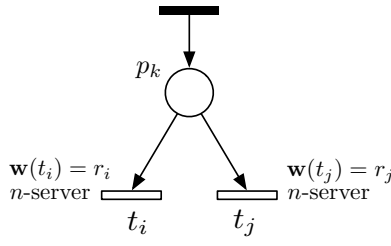

(a) (b)

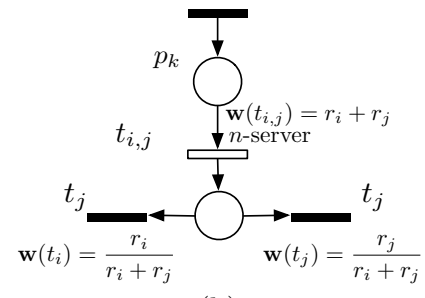

FIGURE 10. A queueing place with more servers: (a) original net; (b) intermediate translation.

queueing policy associated with a place, we follow the general scheme depicted in Fig. 9: in part (a) is the TGSPN where the tagged transitions denoting the arrival and the departure of a customer have been named respectively $t_{\text {arrival }}$ and $t_{\text {departure, whereas the }}$ tagged place subject to scheduling and annotated with the policy has been named $p_{\text {queue; }}$ part (b) shows the duplication needed to treat in a distinguished manner tagged and untagged tokens, together with a schematic representation of the block (subnet) which hides the details of the unfolding that is needed to model the queue implicitly embedded in place $p_{\text {queue }}$ by the TGSPN notation.

In principle, the subnet in the block should explicitly models every position in the queue as well as the modifications occurring there upon each arrival and departure of tokens. Following this approach the size of the subnet would depend on the maximum queue length, which should be specified. In the sequel we propose instead a different and more compact representation of the queue which tracks the position of the tagged customer w.r.t. the other (undistinguishable) customers in the queue. In the following subsections we describe the way in which this block is organized for each of the aforementioned policies. Figure 11 shows the net translations modeling the flows of ordinary and tagged customers through such kinds of queues. For FCFS and LCFS-PR adhoc simpler net translations are provided for the singleserver case although multiple-server translations can always be used.

FCFS scheduling policy. Figure 11(a) shows the GSPN unfolding for the FCFS scheduling policy in the presence of a tagged token. The server of the queue is assumed to be of the $n$-server type. Shown in part (b) of the same figure, the case of a single-service station is treated with a simpler, but equivalent net.

In the net of Fig. 11(a) the whole queue is represented in a compact manner by places $p_{2}, p_{4}$ and $p_{5}$, whereas places $p_{1}, p_{3}$ are for the customers being served, including the tagged one. At each instant, the customers in the queue are distinguished from those arrived before the tagged-customer arrival ("old" customers), modeled by tokens in place $p_{4}$, and those arrived after the tagged-customer arrival ("new" customers), modeled by tokens in place $p_{2}$. A token in place $p_{5}$ represents the tagged-customer waiting at the queue due to server's unavailability. An inhibitor arc $\left(p_{5}, t_{1}\right)$ is used to distinguish among old and new customers and prevents new customers from overtaking the tagged-customer when it is blocked in the queue. On the other hand, old customers waiting for a server to become available prevent the tagged customer to overtake them by means of the inhibitor arc $\left(p_{4}\right.$, req $\left.^{\prime}\right)$. At most $n$ customers are allowed to be served in parallel and the specification of transition $t_{\text {departure }}^{\mathrm{u}}$ of type $n$-server insures the model to incorporate the proper service speed, both when the queue is empty (and thus all the customers in the "queue-place" of the TGSPN are being served) and when the queue is non-empty, but the tagged customer is being served.

The GSPN of Fig. 11(b) is the simplified version when $n=1$. In this case there is no more distinction between customers in queue and in service. Because of this, transition $t_{1}$ has got priority $\left(\pi_{2}\right)$ over $t_{\text {arrival }}^{\mathrm{t}}$ in order to correctly implement a FCFS policy. In fact it is necessary that all the new customers that accumulated in place $p_{2}$ when the tagged customer is either in queue or being served, become immediately "old" as soon as the tagged customer completes its service. Priority $\left(\pi_{2}\right)$ of transition $t_{1}$ prevents the fact that the tagged customer leaving the queue after transition $t_{\text {departure }}^{\mathrm{t}}$ firing, but reentering immediately into the queue for some external and indeterminate reasons via $t_{\text {arrival }}^{\mathrm{t}}$ could overtake customers that are instead ahead of it. Places $p_{q u e u e}^{\mathrm{u}}$ and $p_{\text {queue }}^{\mathrm{t}}$ are introduced in the model for making the equivalence proof of Sec. 6.1 simpler, but are not needed to explain the behavior of the model since they are "implicit".

As an example of the application of this transformation, we can consider the FMS model of Fig. 1, when we are interested in the behavior of the pallets assuming that place $\mathrm{p} 0$ is a queue-place with FCFS policy. Figure 6 depicts the unfolded version of the original model with p0 managed in $\mathrm{RO}$ and the block displayed on the bottom right corner shows how to modify the unfolding of $\mathcal{N}_{\mathbf{f}_{1}}^{+}$, when that same queue-place has a FCFS policy. In this last sub-net, a token in place $p_{0}^{\mathrm{t}}$ represents the tagged pallet at the FCFS queue, whereas places $p_{01}^{\mathrm{u}}$ and $p_{02}^{\mathrm{u}}$ represent the queuing of the other pallets.

LCFS scheduling policy. Figure 11(c) shows the GSPN for the LCFS scheduling policy associated with a tagged queue-place. The transition is assumed to be of type $n$-server.

As for the FCFS case, untagged customers that are not being served are classified as old (tokens in place $p_{3}$ ) or new (tokens in place $p_{1}$ ), being this distinction relevant only if the tagged customer is queueing too. Tokens in place $p_{2}$ and $p_{6}$ model the customers being served with the usual distinction between untagged and tagged entities. Places $p_{1}, p_{3}, p_{5}$ and $p_{7}$ represent the queue.

As soon as the tagged-customer enters the queue 


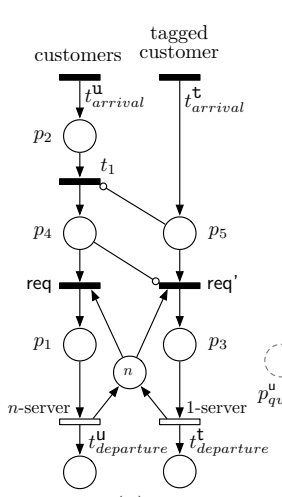

(a)

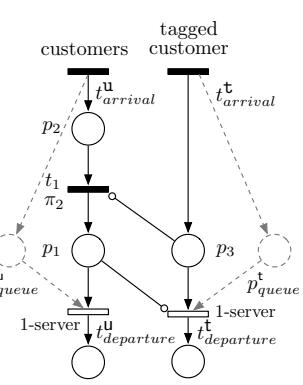

(b)

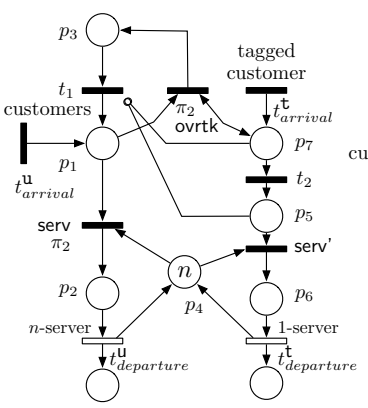

(c)

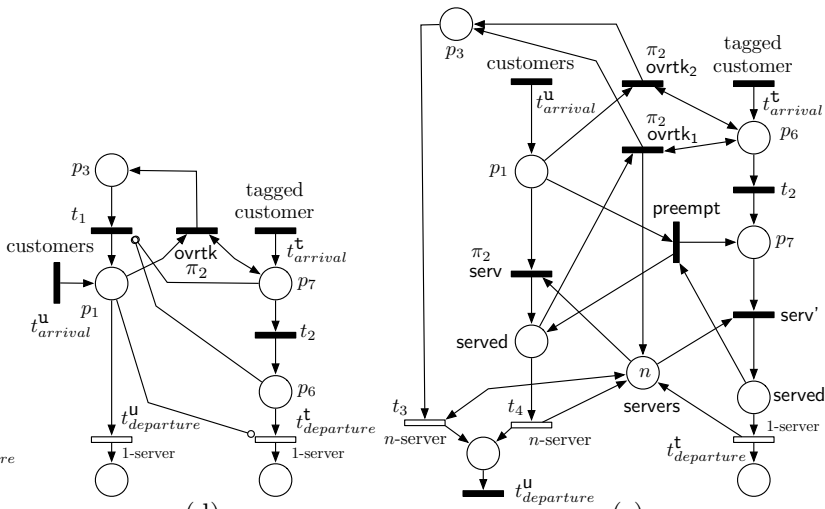

(d) (e)

FIGURE 11. GSPN models of: (a) FCFS with $n$-servers; (b) FCFS with single-server; (c) no preemptive LCFS with $n$-servers; (d) preemptive LCFS with single-server; (e) preemptive LCFS with multiple $n$-servers.

(place $p_{7}$ ) all the customers except those currently being served are moved from $p_{1}$ and put in $p_{3}$ (the higher priority immediate transition ovrtk, implements this action) since they become temporally older than the tagged customer (note that this happens iff serv is not enable): these customers stay in $p_{3}$ till the tagged customer gets the server (tagged token in place $p_{6}$ ), in fact inhibitor arcs $\left(p_{5}, t_{1}\right)$ and $\left(p_{7}, t_{1}\right)$ prevent tokens in $p_{3}$ from leaving this place as long as the tagged token is in the queue. Tokens are put in place $p_{1}$ when new customers arrive. These tokens will overtake the tagged customer possibly blocked in $p_{5}$ as soon as a server becomes available. In the net of Fig. 11(c) this is obtained by assigning priority to transition serv over transition serv'.

LCFS with preemption scheduling policy. The case of a LCFS-PR scheduling policy is more complex than the previous one and we will thus describe the corresponding models starting from the discussion of the single server case first. Figure $11(\mathrm{~d})$ depicts the GSPN model for this initial situation.

The structure of the net is not too different from that of the previous case. The important difference is that the preemption feature makes customers both in queue and in service identical from the point of view of the arriving tagged customer that gets the server in any case. For this reason, untagged customers that joined the queue while the tagged customer was not there, are all represented by tokens in $p_{1}$. When the tagged customer joins the queue, it first makes the other customers already there "old" (a token is put in place $p_{7}$ and the high-priority immediate transition ovrtk changes the status of the customers - if any - already there). When this preliminary action is completed the tagged customer moves to $p_{6}$ and starts being served. If service completes before the arrival of a new untagged customers, $p_{6}$ becomes empty, the old customers move back to $p_{1}$ and one of them is put in service (being they all identical it is not important which of them gets the server). If instead an untagged customer arrives while the tagged one is in service, it preempts it and the new one gets the server. This is represented in the net with the inhibitor arc from $p_{1}$ to $t_{\text {departure }}^{t}$ that interrupts the service of the tagged customer. New untagged customers arriving at the queue in this last situation (i.e., when both $p_{1}$ and $p_{6}$ are non-empty) are simply represented by the addition of new tokens in $p_{1}$ without having to represent explicitly the preemption of untagged customers as they are all identical and their change of state irrelevant from the point of view of the overall behavior of the queue.

When the station is of type $n$-server the net is more complex because of the need to distinguish always between old and new customers. Figure 11(e) shows the GSPN model for this case. To explain how the model works, let's start from the situation in which $n$ untagged customers are being served ( $n$ tokens in place served) and other untagged customers, which were actually arrived before, are in queue (tokens in place $p_{1}$ ). Given the fact that untagged customers are indistinguishable, the overtaking of new untagged customers over old ones (in absence of the tagged customer) does not need to be explicitly represented and simply corresponds to increasing the number of tokens in $p_{1}$.

The arrival of the tagged customer corresponds to a token put in $p_{6}$ whose first effect is that of moving all the tokens corresponding to untagged customers to $p_{3}$. Subsequently, the tagged token moves to place served leaving $n-1$ tokens in place servers. If the tagged customer completes its service before the arrival of a new untagged one, the place servers becomes full again and the subsequent arrivals of untagged customers may yield to the more general situation in which there are $n$ tokens in place served together with other tokens both in places $p_{1}$ and $p_{3}$. The arrival of the tagged token in this situation repeats the sequence of events described before moving all the untagged tokens to $p_{3}$.

Consider now the case in which the tagged customer is being served. First assume that place served is empty and that place servers contains $n-1$ tokens: this means 
that the tagged customer is the last arrived at the queue, independently of the number of tokens in $p_{3}$. A new token in $p_{1}$, corresponding to the arrival of an untagged customer, finds its way to place served because of the high priority of the immediate transition serv. As a consequence, the marking of place servers reduces by one. If this keeps happening, this sequence of actions repeats as long as there are tokens in place servers. At the end, the preemption of the tagged token takes place moving it to place $p_{7}$. New untagged tokens that arrive in this situation get queued in $p_{1}$ and the tagged token re-starts getting service only when all the new tokens move from $p_{1}$ to served and a server becomes available (a token appears in place servers).

Keeping track of the tagged customer as well as of the untagged old and untagged new customers requires the service to be split into three timed transitions (two of type $n$-server for the untagged customers, and one of type single-server for the tagged one) whose firing speeds are controlled (automatically) by their enabling degrees (notice that this is accomplished properly for transition $t_{3}$ due to the test arc connecting to place servers).

\subsection{Models equivalence}

In this section an intuitive explanation is provided, to show that markings of the unfolded model including queue places with scheduling policy different from RO can be mapped to the abstract markings of the untagged GSPN and that properties similar to those proved in Sec. 5 for the restricted case of the RO scheduling policy hold also in this case.

For the sake of simplicity let us refer to the case of FCFS scheduling policy in a queue place with only one single server timed output transition Its unfolding is shown in Fig. 11.b. Observe that places $p_{q u e u e}^{\mathrm{t}}$ and $p_{\text {queue }}^{\mathrm{u}}$ are implicit places, that is their marking can be deduced from the marking of other places in the net (in the example $\mathbf{m}\left(p_{\text {queue }}^{\mathrm{t}}\right)=\mathbf{m}\left(p_{3}\right)$ and $\mathbf{m}\left(p_{\text {queue }}^{\mathrm{u}}\right)=$ $\left.\mathbf{m}\left(p_{1}\right)+\mathbf{m}\left(p_{2}\right)\right)$, and they never constrain the enabling of a transition (if a transition that has either $p_{\text {queue }}^{\mathrm{t}}$ or $p_{\text {queue }}^{\mathrm{u}}$ in its input set is not enabled, then there is at least another place which prevents its enabling). These two places can be seen as counters indicating the total number of tagged/untagged tokens in the queue, independently of their position. The mapping of the refined states into more abstract (untagged) states with respect to place $p_{\text {queue }}$ is the same as it was for the RO scheduling policy $\left(\mathbf{m}\left(p_{\text {queue }}\right)=\right.$ $\left.\mathbf{m}^{\prime}\left(p_{\text {queue }}^{\mathrm{t}}\right)+\mathbf{m}^{\prime}\left(p_{\text {queue }}^{\mathrm{u}}\right)\right)$, however in this case the number of "refined" states that are contained in an aggregate is larger, because it accounts also for the relative position of the tagged customer in the queue. It is possible to prove that the following statements hold:

- if $\mathbf{m}\left(p_{\text {queue }}^{\mathrm{t}}\right)>0 \wedge \mathbf{m}\left(p_{\text {queue }}^{\mathrm{u}}\right)=0, t_{\text {departure }}^{\mathrm{t}}$ is enabled, and hence the behavior is the same as in the case of the RO scheduling policy;
- if $\mathbf{m}\left(p_{\text {queue }}^{\mathrm{t}}\right)=0 \wedge \mathbf{m}\left(p_{\text {queue }}^{\mathrm{u}}\right)>0, t_{\text {departure }}^{\mathrm{u}}$ is enabled, and hence the behavior is the same as in the case of the RO scheduling policy;

- if $\mathbf{m}\left(p_{\text {queue }}^{\mathrm{t}}\right)>0 \wedge \mathbf{m}\left(p_{\text {queue }}^{\mathrm{u}}\right)>0$ either $t_{\text {departure }}^{\mathrm{u}}$ or $t_{\text {departure }}^{\mathrm{t}}$ is enabled (but not both), depending on the relative position of the tagged token in the queue

Observe that in the first two cases the behavior is equivalent to that of then RO scheduling policy (where we have seen that the MC associated with the tagged version is lumpable into the MC corresponding to the untagged one), and hence the proof of equivalence is similar to that presented in Sec. 5.2. In the last case there is a difference: in the RO unfolded model both transitions are enabled and are in race. The global rate is marking dependent ensuring that the sum of the two rates is $w\left(t_{\text {departure }}\right)$. In the FCFS case, the two transitions (which have the same rate) are never enabled together. In any case the state that is reached after the firing of either $t_{\text {departure }}^{\mathrm{u}}$ or $t_{\text {departure }}^{\mathrm{t}}$ belongs to the same aggregate, hence the lumpability condition is satisfied. Similar considerations hold for the other policies.

The sequences of immediate transitions "internal" to each policy-specific unfolded submodel that are activated by each arrival to (departure from) the queue place are deterministic, since the new configuration of the queue is uniquely determined by the scheduling policy, hence they do not modify the rate of the timed transition associated with such path.

\section{COMPUTING THE PASSAGE TIME OF THE UNFOLDED TGSPN}

Let $\mathcal{N}$ and $\mathcal{N}^{\prime}$ be respectively the untagged GSPN and its unfolding, both derived from the TGSPN(see Sec. 5). The computation of the first passage time involves the following steps:

1. Generation of the $\mathrm{MC}_{\mathcal{N}^{\prime}}$ of the unfolded GSPN $\mathcal{N}^{\prime}$. 2. Selection of the start, end and forbidden states according to the definition of sets ENTRY, EXIT and $F O R B I D$, as explained in details in the final paragraph of this section.

3. Computation of the first passage time distribution through classical techniques [13]: this requires to make all the end and forbidden states in the CTMC absorbing (i.e., all transition rates out of these states must be set to 0). Moreover, when there are several start states, an initial probability distribution over these states must be provided to combine through a weighted sum the (usually different) passage time distributions obtained for each possible start state.

The start states probability distribution can be provided in different ways: (1) it can be derived from the Embedded Markov Chain steady state distribution (when it exists), renormalized over the subset of start states, (2) it can be derived from the transient states probability distribution at time $t$ (again, renormalized 

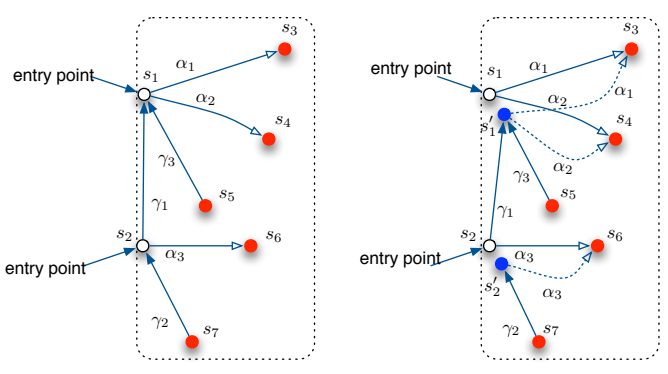

FIGURE 12. Replication of starting states $s_{1}$ and $s_{2}$ into $s_{1}^{\prime}$ and $s_{2}^{\prime}$ due to internal reachability.

over the set of start states), (3) it can be a user-defined initial distribution, specified by the modeler depending on the objectives of the study. If the first option is chosen, it is possible to optimize this step by computing the steady state probability of the $\mathrm{MC}_{\mathcal{N}}$ before applying the unfolding, and then properly distributing it to the refined start states of $\mathrm{MC}_{\mathcal{N}^{\prime}}$ : this optimization is described in detail later in this section.

If the start states probability distribution is obtained from the EMC steady state distribution, attention should be paid to those start states that, besides being reachable through entry points, can also be reached via the firing of other transitions. In this case in fact, the state should be replicated into two states, one reached only by proper "entry point" arcs, and one reached by all other arcs. The two copies of the state will have identical output transitions, but only the first copy is considered as a start state. The steady state probability should be computed after this transformation (we denote $\mathrm{MC}_{\mathcal{N}^{\prime}}^{+}$the corresponding $\mathrm{MC})$. Fig. 12 illustrates the procedure on a pair of starting states, $s_{1}$ and $s_{2}$, that besides being reachable through proper entry points, are also reachable from states $s_{5}$ and $s_{7}$ through transitions that are not entry points. States $s_{1}^{\prime}$ and $s_{2}^{\prime}$ are added, with output arcs that are copies of the output arcs of the original $s_{1}$ and $s_{2}$ states, respectively, and the transitions from $s_{5}$ and $s_{7}$ are redirected towards these new states. It is easy to see that the new chain is lumpable into the original one, but it allows to correctly evaluate the relative frequency of reaching each start state through an entry point. A situation of this kind happens in the model of Fig. 4 (e.g. the start state can be reached again if a failure happens in the first stage of the service, and then a repair brings the system back into the same state).

Several tools are available to compute the passage time distribution, given the CTMC with absorbing end and forbidden states and an appropriate start states probability distribution (e.g. [6, 7]). In Sec. 8 some results on the running example obtained with a prototype implementation are illustrated. Work is in progress to interface our implementation of the first two steps with the very efficient algorithms for passage time distribution computation implemented in Hydra [7].
Identification of start, end, and forbidden states. Starting from the net level definition of entry, exit and forbidden points (events), identifying a traversal subnet $\mathcal{N}_{\mathcal{S}}$, coupled with conditions on markings, the derivation of the start, end and forbidden states of the CTMC can be performed transparently to the modeler. A reachable (tangible) marking $\mathbf{m}_{s}$ is a start state iff it is reachable from a tangible marking $\mathbf{m}^{\prime}$ through the firing of a sequence of one timed and zero or more immediate transitions containing one transition $t_{j}^{\mathrm{t}}$ such that:

- $\left\langle t, C_{t}^{\text {pre }}, C_{t}^{\text {post }}\right\rangle \in E N T R Y$, and the firing of $t_{j}^{\mathrm{t}}$ moves the tagged token from place $p_{j}^{\mathrm{t}}$ to place $p_{k}^{\mathrm{t}}$, and $p_{j} \notin \mathcal{S}$ while $p_{k} \in \mathcal{S}$,

- and $C_{t}^{\text {post }}\left(\mathbf{m}_{s}\right)=$ true $\wedge C_{t}^{\text {pre }}\left(\mathbf{m}^{\prime}\right)=$ true;

observe that the tagged token must be outside $\mathcal{S}$ in $\mathbf{m}^{\prime}$ while it is inside $\mathcal{N}_{\mathcal{S}}$ in $\mathbf{m}_{s}$ (these two conditions might be embedded into $C_{t}^{\text {pre }}$ and $C_{t}^{\text {post }}$ respectively). Observe that conditions $C_{t}^{\text {pre }}$ and $C_{t}^{\text {post }}$ refer to places in the TGSPN (whose marking can be retrieved from their tagged and untagged copies in the unfolded GSPN), but can also refer to the presence (or absence) of the tagged token in a given place of the TGSPN (which translates in a condition on the marking of the tagged copy of the place).

An end state is any (tangible) marking $\mathbf{m}_{e}$ reachable from a start state $\mathbf{m}_{s}$ through a firing sequence $\sigma$ such that: $\sigma=\sigma^{\prime}+\sigma^{\prime \prime}, \mathbf{m}_{s} \stackrel{\sigma^{\prime}}{\rightarrow} \mathbf{m}^{\prime} \stackrel{\sigma^{\prime \prime}}{\rightarrow} \mathbf{m}_{e}$, where $\sigma^{\prime \prime}$ is a sequence of one timed transition and zero or more immediate transitions, including (a tagged copy $t_{j}^{\mathrm{t}}$ of) one exit point $t \in \mathcal{S}_{\text {out }}$, moving the tagged token from one place inside $\mathcal{S}$ to one place outside $\mathcal{S}$, hence the tagged token is outside $\mathcal{N}_{\mathcal{S}}$ in $\mathbf{m}_{e}$ while it is inside $\mathcal{N}_{\mathcal{S}}$ in $\mathbf{m}^{\prime}$. Also in this case the conditions $C_{t}^{\text {pre }}$ and $C_{t}^{\text {post }}$ associated with $t$ in set EXIT must be satisfied respectively by $\mathbf{m}^{\prime}$ and $\mathbf{m}_{e}$. A similar definition holds for forbidden states.

Optimizing the start states distribution computation The computation of the steady state distribution of the start states could be performed more efficiently if some conditions hold: the idea is to derive such probability distribution from that of the abstract states of the GSPN $\mathcal{N}$, instead of that of the refined GSPN $\mathcal{N}^{\prime}$. Given any abstract marking $\widehat{\mathbf{m}}$ it is possible to derive all the refined markings $\mathbf{m}_{i}$ (characterized by $\left.\mathbf{m}_{i}\left(p_{i}^{\mathrm{t}}\right)=1, \mathbf{m}_{i}\left(p_{i}^{\mathrm{u}}\right)=\widehat{\mathbf{m}}\left(p_{i}\right)-1\right)$ that it represents, and using combinatorial arguments, compute the steady state distribution of such refined marking as follows: $\pi\left(\mathbf{m}_{i}\right)=\pi(\widehat{\mathbf{m}}) \widehat{\mathbf{m}}\left(p_{i}\right) / K$ where $K=\mathbf{f} \cdot \mathbf{m}_{0}$ (see Sec. 2). Intuitively this can be explained as follows: the tagged token behaves exactly as the untagged ones, hence any token in one of the tagged places of $\widehat{\mathbf{m}}$ has equal probability to be chosen as the tagged one; as a consequence the probability that the tagged token is in one specific tagged place is proportional to the number of "taggable" tokens contained in it.

One may even think to avoid building the whole 
$R G\left(\mathcal{N}^{\prime}\right)$, and instead derive only that part of it which is needed for the computation of the measure of interest: the relevant start states could be generated by inspecting the smaller $R G(\mathcal{N})$. This optimization however can be applied only if all the refined states of any reachable abstract marking $\widehat{\mathbf{m}}$ are reachable too: this may not be true, depending on the initial marking, and the initial position of the tagged token. A sufficient condition for this property to hold is that the $R G(\mathcal{N})$ is strongly connected and there exists a reachable abstract marking $\widehat{\mathbf{m}}$ such that all customers (tokens) are in the same tagged place $p_{j}$ (the running example satisfies this condition in its initial marking).

\section{EXPERIMENTAL RESULTS}

In this section some experimental results are presented which have been computed with a prototype implementation based on GreatSPN [14] for the generation of the CTMC, and on an algorithm written for Octave and used for the computation of the first passage time distributions. GreatSPN is also used to construct the sets of start, end, and forbidden states, and for the computation of the (steady state) initial distribution of the start states. The prototype has some limitation on the size of the models that it can treat, but our aim is to eventually interface the model generation part with some existing efficient passage time computation implementation (e.g. Hydra [7]).

The two measures specified in Sec. 4 have been computed for the running example of Fig 1: the first one measures the time from the entrance of the tagged token through the entry point $T_{4}$ up to its exit point $T_{2}$ (traversal of $\mathrm{L} / \mathrm{U}$ station and of machines M1 and M2). The analysis has been repeated assuming different scheduling policies of place $p 0$ (queue in front of the $\mathrm{L} / \mathrm{U}$ station). Three policies have been considered: FCFS, LCFS-PR and RO. The second measure that we have considered is the duration of a repair action performed by a repairman in the period that lasts between two subsequent vacations: in this case the entry point is $T 9$ while the exit point is $t 7$. Notice that two different TGSPNs are defined in the two cases, since two different p-semiflows are considered for these two cases to identify the class of customers of interest, hence two different unfolded models are used to obtain the result in the two cases (producing refined RGs of different size). The timed transitions are all of type single server, moreover $w(T 0)=0.7$, $w(T 2)=2.0, w(T 3)=3.0, w(T 4)=4.0$, while all other timed and immediate transitions have weight equal to 1.0. The considered scenario has five pallets, two repairmen and only one spare tool (for machine $M 2$ ). In Fig. 13 the Cumulative Distribution Function (CDF) of the first measure concerning the pallets is shown: as expected the CDFs of the first passage time for the three scheduling policies are different, while they all have the same first moment that turns out to be equal

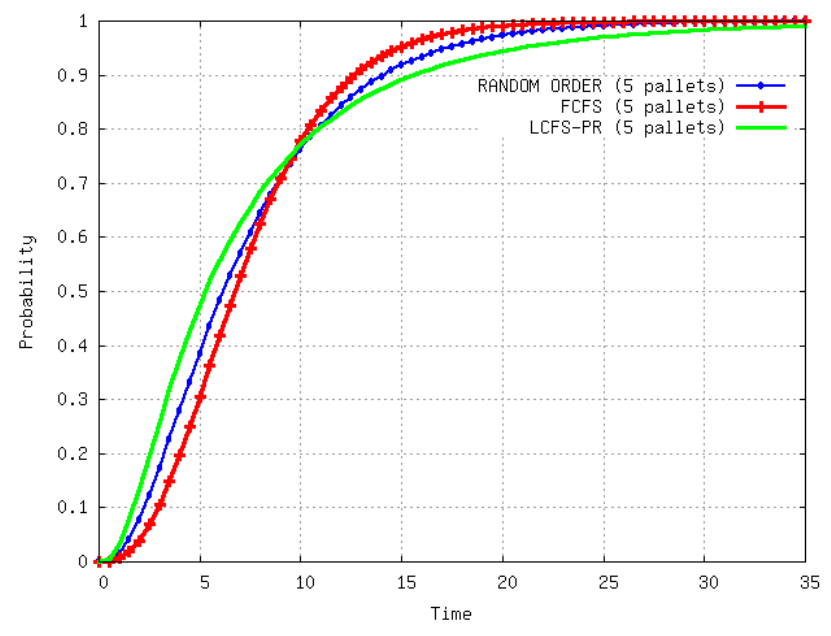

FIGURE 13. CDF of the first passage time of a pallet through the $\mathrm{L} / \mathrm{U}$ station and the manufacturing stations $M 1$ and M2, with different scheduling policies at the $\mathrm{L} / \mathrm{U}$ input queue.

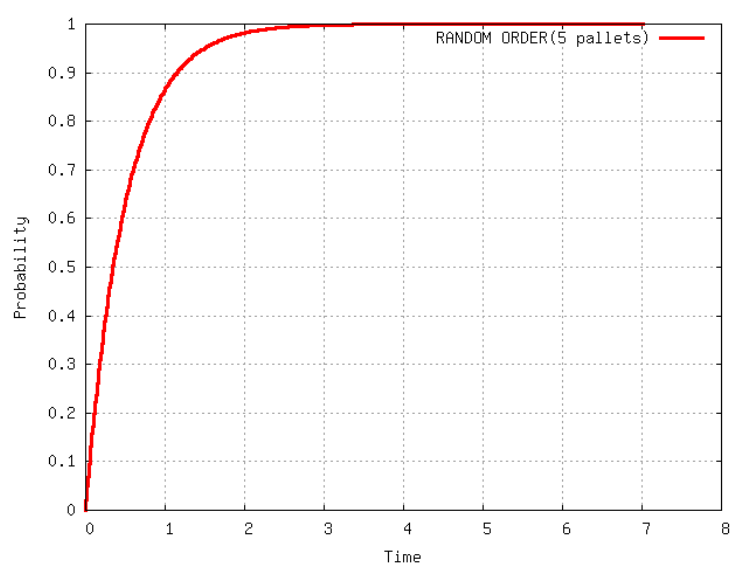

FIGURE 14. CDF of the first passage time from the beginning of a repairman working cycle to the end of a repairing cycle without taking any intermediate vacation.

to 7.3958 for all scheduling policies, and coincides with the average time required for a (pallet) token to traverse the considered net, computed from the steady state distribution of the states in the more abstract model (applying Little's formula). The difference among the three CDFs becomes more relevant as the number of clients in the system increases, and as the rate of transition TO becomes lower (corresponding to a slow down in the $\mathrm{L} / \mathrm{U}$ station).

In Fig. 14 the CDF of the first passage time from the beginning of a repairman working cycle to the end of a repairing cycle (without taking any intermediate vacation) is shown (the rate and marking parameters are the same as in the previous example); The value of the first moment is 0.500006 , and coincides with the average value computed on the more abstract GSPN. 


\section{DISCUSSION OF RELATED WORK}

The ideas of using a tagging procedure to compute first passage times in GSPNs have been originally presented in [2], which is the first paper addressing this topic in a systematic manner; earlier works developing similar ideas on various formalisms [5, 6], in general provide less support to the modeler and require a deeper knowledge of the underlying Markov Chain.

The first substantial difference of our proposal w.r.t. [2] concerns the way used to choose the places and the transitions to be tagged, that we base on the computation of the p-semiflows of the net which identify the locations where tokens are preserved and thus "circuits" where the movement of tokens can be interpreted as a flow of customers. This choice overcomes a potential source of problems contained in the definition proposed in [2] where their restrictions do not seem to require that either none or all the arcs departing from a (tagged) place must be tagged. We believe that without this restriction it is not possible to guarantee that the behavior of the untagged GSPN is "equivalent" to (i.e. is a lumping of) that of the unfolding of the tagged GSPN. In Fig. 15 two

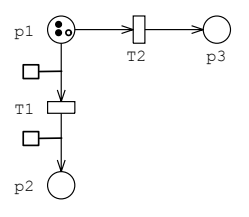

(a)

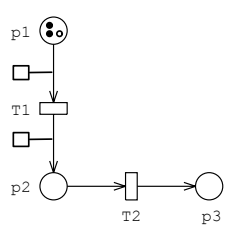

(b)
FIGURE 15. Two situations where the tagged token behavior is different from that of the other tokens.

examples of these problems are given where the tagging is specified according to the notation proposed into [2]: in (a) the tagged token can only flow in one of the two alternative branches, hence the marking where all tokens reach place $p 3$ is unreachable in the tagged model, while it is reachable in the untagged one. In (b) the tagged token remains blocked into place $p 2$, without possibility of proceeding further. In both cases the tagged model behavior may not be equivalent to that of the untagged ones: this seems to potentially introduce a bias in the computation of the first passage time that is avoided with our approach.

From the point of view of the specification of start and end states, [8] and [2] propose a method that is purely based on the definition of predicates on the markings; our definition is instead event-centered (similar to the "probes" approach proposed in [5]), and has been devised to be more intuitive for the modeler. In fact, we believe that is often easier to indicate the start of a path subject to measure by means of an event (transition firing, typically representing the entrance into a subnet), possibly enriched with an additional condition on the marking. A specification purely based on the marking characterization is surely possible, but requires the modeler to have a complete and precise view of the states truly corresponding to the entrance in a given subnet (which may not be trivial, in particular when immediate transitions are involved).

Concerning the way of obtaining the unfolded model from the tagged one, the proposal in [2] is based on the Colored GSPNs (CGSPNs): in our opinion this is a very promising idea to follow, however in this case applying the unfolding is not the only possible option. In fact the RG and the corresponding CTMC can be directly derived for the CGSPN applying the firing rule specific of this formalism.

Finally, for what concerns the introduction of scheduling policies associated with queue places, the literature contains the proposal of a Queuing Petri Net (QPN) formalism $[9,10]$ which allows to embed queuing places in Colored GSPN models, thus extending the classical GSPN formalism. In QPNs, queuing places embed the actual queue, the servers and a sort of "output buffer" called "depository" from which tokens that have already been served can be withdrawn by transitions (in [10] immediate queuing places are also included). Our approach is somehow different since we provide an original and efficient way to consider scheduling policies different from the random order one, where an interesting feature is the compact and parametric representation of the queue, specifically tailored to the goal of tracking the tagged token position in the queue. The semantics of TGSPNs with queue places is formally defined by means of an unfolding and queue place substitution procedure, that allows to automatically generate a GSPN model, ready for generation of a CTMC on which existing passage time density computation techniques can be applied. Since our base model is GSPN rather than CGSPN, the only identities to be distinguished are thus untagged and tagged tokens, and only one tagged token is allowed in the net, the modeling of the queue can be simplified and made parametric (i.e., the structure of the net is kept independent on the maximum number of "customers" arriving at the queue).

\section{CONCLUSION AND FUTURE WORK}

In this paper a new definition of Tagged GSPN has been introduced, in which tagged places and transitions are chosen on the basis of methods that exploit the structural properties of the model.

An important extension is the proposal of an original and efficient way of considering several scheduling policies for the management of the tokens in the input places of transitions; compact and parametric representations of these policies are presented.

A formal proof is provided to show that the unfolded model where the tagged customers and the queue policies are explicitly represented is equivalent to the original one.

Finally, thanks to a prototype implementation of 
our approach in the GreatSPN framework, we have performed and reported some experimental results, which highlighted the importance of distinguishing among different scheduling policies for the correct computation of the first passage time distributions.

There are several possible developments of the work presented in this paper, mostly related with the use of High Level Petri Net formalisms. Indeed, it will be interesting to extend the approach that we propose in this paper to HLSPNs since colors could be used not only for tagging a specific customer, but also for a more compact modeling of other situations where distinguished identities may be useful (e.g. to represent different classes of customers, with different service time or scheduling priority, or to correctly model forkjoin behaviors where the join must synchronize the subprocesses of the same originating process). A formalism that is very well suited for this purpose is that of Stochastic Well-Formed Nets (SWN): the Symbolic Reachability Graph (SRG) algorithm can be exploited in this case to adapt the abstraction level to be used for the analysis [15]. The running example considered in this paper can be easily transformed in a SWN by using colors to distinguish all customer tokens with unique identifiers. The SWN syntax allows to explicitly specify which colors lead to a distinguished behavior, and which instead are assigned to tokens with homogeneous behavior: if all colored customers have homogeneous behavior, the SRG automatically adapts its size to exploit such symmetry, and in our example has the same size as the untagged GSPN RG. If one color is separated from the others (in SWNs this can be done by partitioning a color class into static subclasses), then the SRG has the same size (and structure) as the RG of the unfolded GSPN. Colored transition rates and weights adapt to the number of transition instances involving tokens with different colors: this on one hand avoids the need to specify marking dependent weights (e.g. when performing a choice between firing a transition for the tagged or one untagged customer) but on the other hand requires to properly handle transitions representing service centers with single server. There are cases in which using "black" tokens is more practical from the modeling point of view, for example when groups of tokens need to be "merged" into a single token (a situation that leads to p-semiflows where some places have a weight greater than one). Finally, the SRG computation costs more than the RG computation, so that the choice between using the method proposed in this paper or a new method based on the SWN formalism may depend on the system to be modeled and should be carefully evaluated to find a good trade off.

\section{REFERENCES}

[1] Marsan, M. A., Balbo, G., Conte, G., Donatelli, S., and Franceschinis, G. (1995) Modelling with Generalized
Stochastic Petri Nets. J. Wiley, New York, NY, USA.

[2] Dingle, N. J. and Knottenbelt, W. J. (2009) Automated Customer-Centric Performance Analysis of Generalised Stochastic Petri Nets Using Tagged Tokens. Electron. Notes Theor. Comput. Sci., 232, 75-88.

[3] Nelson, R. (1995) Probability, Stochastic Processes, and Queueing Theory: The Mathematics of Computer Perf. Eval. Springer - Verlag, New York, NY,USA.

[4] Miner, A. S. (2003) Computing response time distributions using stochastic Petri nets and matrix diagrams. $10^{\text {th }}$ Int. Workshop on Petri Nets and Performance Models (PNPM'03), Urbana-Champaign, IL, USA, Sept, pp. 10-19. IEEE Comp. Soc.

[5] Bradley, J. T., Dingle, N. J., Gilmore, S. T., and Knottenbelt, W. J. (2003) Derivation of Passage-time Densities in PEPA Models using ipc: the Imperial PEPA Compiler. $11^{\text {th }}$ Int. Symp. on Modeling, Analysis, and Simulation of Computer Systems, Los Alamitos, CA, USA, pp. 344-345. IEEE Comp. Soc.

[6] Bodrog, L., Horvath, G., Racz, S., and Telek, M. (2006) A tool support for automatic analysis based on the tagged customer approach. $3^{\text {rd }}$ Int. Conf. on the Quantitative Evaluation of Systems '06, Riverside, CA, USA, Sept, pp. 323-332. IEEE Comp. Soc.

[7] Dingle, N. J., Harrison, P. G., and Knottenbelt, W. J. (2004) Uniformisation and Hypergraph Partitioning for the Distributed Computation of Response Time Densities in Very Large Markov Models. Journal of Parallel and Distributed Computing, 64(8), 309-920.

[8] Suto, T., Bradley, J. T., and Knottenbelt, W. J. (2007) Performance trees: Expressiveness and quantitative semantics. $4^{\text {th }}$ Int. Conf. on Quantitative Evaluation of Systems (QESTO7), Edinburgh, UK, Sep., pp. 4150. IEEE Comp. Soc.

[9] Bause, F. and Kritzinger, P. F. (Second Edition, 2002) Stochastic Petri Nets - An Introduction to the Theory. Friedr. Vieweg \& Sohn Verlag, Braunschweig/Wiesbaden, Germany.

[10] Bause F. (1993) Queueing Petri Nets - A formalism for the combined qualitative and quantitative analysis of systems. $5^{\text {th }}$ Int. Workshop on Petri Nets and Performance Models, Toulouse,France, Oct., pp. 14-23. IEEE Comp. Soc.

[11] Balbo, G., De Pierro, M., and Franceschinis, G. (2009) Tagged Generalized Stochastic Petri Nets. In Bradley, J. T. (ed.), 6th European Perf. Eng. Workshop, EPEW, London, UK, July, LNCS, 5652, pp. 1-15. Springer.

[12] Teruel, E., Franceschinis, G., and De Pierro, M. (2003) Well-Defined Generalized Stochastic Petri Nets: A NetLevel Method to Specify Priorities. IEEE Trans. Softw. Eng., 29, 962-973.

[13] Kulkarni, V. (1995) Modeling and Analysis of Stochastic Systems. Chapman \& Hall, London, UK.

[14] Baarir, S., Beccuti, M., Cerotti, D., De Pierro, M., Donatelli, S., and Franceschinis, G. (2009) The GreatSPN tool: recent enhancements. SIGMETRICS Performance Evaluation Review, Special Issue on Tools for Performance Evaluation, 36, 4-9.

[15] Chiola, G., Dutheillet, C., Franceschinis, G., and Haddad, S. (1993) Stochastic well-formed coloured nets for symmetric modelling applications. IEEE Trans. on Computers, 42, 1343-1360. 